\title{
From Mind to Market: A Global, Dynamic Analysis of R\&D*
}

\author{
Jeroen Hinloopen $^{\dagger} \quad$ Grega Smrkolj $^{\dagger \dagger} \quad$ Florian Wagener ${ }^{\dagger}$
}

September, 2011

\begin{abstract}
Existing models of R\&D are not easily reconciled with four observable aspects of R\&D: initial technologies ("ideas") need to be developed further, only a minority of initial ideas is successfully brought to the market, production and process innovations take place simultaneously (whereby, initially, there is no production at all), and process innovations are implemented for technologies that are destined to leave the market. We present a detailed bifurcation analysis for a dynamic model of $R \& D$ that captures these observations in one, unifying framework. As we provide a global analysis, we do not limit initial technologies to carry marginal costs that are below the choke price. We show that there always exists a critical value of initial marginal cost above which the firm does not initiate any (R\&D) activity; the saddle-point steady state is never globally optimal. We also sketch some policy implications of our analysis.
\end{abstract}

Keywords: Bifurcations, Innovation, Monopoly, Optimal control, R\&D

JEL: D42, L12, O31

\section{Introduction}

Around 1900, Swiss chemist Jacques E. Brandenburger invented a transparent film which he named "cellophane". On December 26, 1923, American chemical company DuPont acquired from La Cellophane Société Anonyme, an organization to which Brandenburger had assigned his various patents, the exclusive rights to its United States cellophane patents. DuPont's hopes for creating a lucrative market were shattered, however, by discovering that cellophane could not be used to wrap up products that

\footnotetext{
*The authors would like to thank Aufa Doarest, Bruno Cassiman, Myong-Hun Chang, Herbert Dawid, Flóra Felsö, Dieter Grass, Kenneth Judd, Tatiana Kiseleva, Reinhilde Veugelers, and participants at the Tinbergen Institute seminar (Rotterdam, May 2010), at the 11th Workshop on Optimal Control, Dynamic Games and Nonlinear Dynamics (Amsterdam, June 2010), at EARIE 2010 (Istanbul, September 2010), at the 5th EBIM Doctoral Workshop on Economic Theory (Bielefeld, November 2010), at IIOC 2011 (Boston, April 2011), at the CISS 2011 Summer School (Turunc, May 2011), and at EEA-ESEM 2011 (Oslo, August 2011) for many valuable comments and discussions. Any remaining errors are those of the authors.

${ }^{\dagger}$ University of Amsterdam and Tinbergen Institute

${ }^{\ddagger}$ Corresponding author. University of Amsterdam, Amsterdam School of Economics, Roetersstraat 11, 1018 WB Amsterdam, The Netherlands. E-mail address: g.smrkolj@uva.nl
} 
require moisture proofing, such as cake and candy. It took DuPont chemist William H. Charch three years and thousands of tests to develop a lacquer that made cellophane moisture proof, an invention that revolutionized the packaging and merchandizing industry. As the manufacturing costs of cellophane continued to decline due to DuPont's ongoing process innovations, so did the prices of cellophane. All this contributed to cellophane being used as wrapping material for a variety of products (from food to jewelry), and for its use in various products (including batteries, scotch tape, and dialysis machines). Since the mid-1930's, cellophane has been manufactured continuously. It is still used today.

The development of cellophane, from mind to market, is typical for the life cycle of many new technologies. Research starts long before a prototype sees the light; development begins long before the launch of a new product. However, ideas abound, but only a small fraction leads to successful innovations. For instance, in 1979, Apple was enthusiastic about a design for a computer mouse that they had discovered at Xerox research center. But Apple did not develop this prototype further as the projected production costs of a single mouse would be over $\$ 400 .{ }^{1}$ Also, existing technologies tend to leave markets slowly due to incumbents' R\&D efforts. For example, Edison's invention of an electric light bulb was bound to replace the gas lamp. Producers of the latter however prolonged the lifetime of this inferior technology through continuous product innovations, including the introduction of the Welsbach mantle that made gas lamps five times more efficient (Utterback, 1994).

These examples illustrate four stylized facts of R\&D: (i) initial technologies ("ideas" or "prototypes") need to be developed further before they are suitable for large-scale production; (ii) there are many initial technologies, but only a very limited fraction is developed further, and from this fraction only a subset will constitute a successful innovation, (iii) production and the search for further improvements take place simultaneously, whereby production starts only after an initial stage of successful product development, and (iv) there are process innovations for technologies that are destined to leave the market in the foreseeable future.

Existing models of R\&D are not easily reconciled with these observations. Static models of R\&D are silent about the process from prototype to first releases of new products and production technologies. ${ }^{2}$ Moreover, these models are forced to assume that marginal costs, the proxy for production technologies, are below the choke price. This assumption is quite unlikely to hold for new technologies in their early stages of development.

Dynamic models of R\&D are in principle equipped to capture the path from prototype to market penetration. To date, however, neither "innovation race" models nor

\footnotetext{
${ }^{1}$ Years later, Apple came up with a new design which would only cost $\$ 25$ to produce. This prototype was subsequently developed into Apple's famous single-button mouse.

${ }^{2}$ The seminal papers in this literature are d'Aspremont and Jacquemin (1988) and Kamien et al. (1992); see De Bondt (1997) for an overview.
} 
dynamic versions of conceived static models do so adequately. In essence, models of innovation races examine the time of completion of a cost-saving innovation of known magnitude, whereby the expected time of completion is one-to-one related to R\&D expenditures. ${ }^{3}$ These models exclude the coexistence of production and R\&D efforts. Moreover, the $R \& D$ process cannot fail, thus transforming the $R \& D$ investment decision into a static one. The recently conceived dynamic versions of static R\&D models maintain the assumption that marginal costs are below the choke price at all times. ${ }^{4}$ Initial technologies that are "expensive" are excluded, by assumption, from the analysis. Hence, R\&D efforts always lead to the stable equilibrium. Put differently, every initial technology will be developed further, and successfully so. Indeed, without exception this literature provides analyses that are locally optimal only.

In this paper, we provide a generalized description of the economics of R\&D. In particular, for any initial technology level $c_{0}$, we characterize for a monopolist the investment and production path that is globally optimal. Hence, we do not exclude initial technologies that are above the choke price, and we do not restrict ourselves to solutions that are only locally optimal. ${ }^{5}$ Our model is, therefore, more easily reconciled with the four stylized facts of R\&D mentioned earlier. ${ }^{6}$

From a technical point of view, the problem of the monopolist is formulated as an optimal control problem with an infinite time horizon. Its distinct characteristic is the presence of multiple equilibria while at the same time the Arrow-Mangasarian sufficiency conditions are not met. In models of this type, the qualitative properties of optimal solutions may change if parameters are varied. We therefore use bifurcation analysis ${ }^{7}$, which constitutes the mathematics of a qualitative change, to assess industry dynamics for all initial technologies in conjunction with other parameter combinations (including time preferences and the efficiency of the R\&D process).

Our analysis yields four distinct scenario's, three of which remain hidden in local analyses: (i) initial marginal costs are above the choke price and the $R \& D$ process is initiated; after some time production starts and marginal costs continue to fall with subsequent R\&D investments; (ii) initial marginal costs are above the choke price and the R\&D process is not initiated, yielding no production at all; (iii) initial marginal costs are below the choke price and the R\&D process is initiated; production starts

\footnotetext{
${ }^{3}$ Seminal contributions here include Loury (1979) and Lee and Wilde (1980). Reinganum (1989) surveys this literature.

${ }^{4}$ This literature is still scant; it includes Cellini and Lambertini (2009), Lambertini and Mantovani (2009), and Kováč et al. (2010).

${ }^{5}$ As it turns out, a non-trivial part of the parameter space yields stable paths with initial marginal costs so high that production would yield negative mark-ups. At that stage all the monopolist does is to invest in $\mathrm{R} \& \mathrm{D}$ in order to bring down the costs of production.

${ }^{6}$ As this is the first analysis ever to consider the entire range of initial technologies, we restrict ourselves to the monopoly case. Obviously, competitive forces play an important role as well in what we observe about R\&D.

${ }^{7}$ Variations in parameter values typically lead to qualitative changes in the solution structure (e.g., some steady states lose their stability, indifference points appear, and so on). Such qualitative changes in the solution structure due to smooth variations in parameters are called bifurcations (see Grass et al. (2008)).
} 
immediately and marginal costs continue to fall over time, and (iv) initial marginal costs are below the choke price and the initiated $R \& D$ process is progressively scaled down; production starts immediately but the technology (and production) will die out over time; the firm leaves the market. The strength of our analysis is that all these cases can emerge from the same unifying framework.

The analysis is not without policy implications. It shows that market characteristics that affect future profitability have an impact on the monopolist's decision to develop further an initial technology. If market regulations are such that future mark-ups are reduced a priori, it could be that the range of initial technologies that is developed further will shrink. The loss of total surplus this reduction brings about constitutes a hidden cost of market regulations if the initial technology consists of marginal costs above the choke price. In that case, there is no immediate production lost if the initial technology is not developed further. Yet, these indirect costs should be taken into account when assessing properly the trade-off between static and dynamic efficiency.

The rest of the paper is organized as follows. The model is introduced in the next section and its steady states are characterized in Section 3. The properties of the global equilibrium and corresponding industry dynamics are considered in Section 4. Section 5 concludes.

\section{The model}

Time $t$ is continuous: $t \in[0, \infty)$. A single supplier produces at marginal costs $c(t)$. In every instant, market demand equals production

$$
q(t)=A-p(t)
$$

where $p(t)$ is the market price, $q(t)$ is the quantity produced, and $A$ is the choke price. The monopolist obtains an initial, exogenous technology $c(0)=c_{0}$. He can reduce this marginal costs by exerting $\mathrm{R} \& \mathrm{D}$ effort $k(t)$. Marginal costs evolve as follows over time: ${ }^{8}$

$$
\frac{d c(t)}{d t} \equiv \dot{c}(t)=c(t)(-k(t)+\delta)
$$

\footnotetext{
${ }^{8}$ One interpretation of the model is that $c_{0}$ constitutes the initial level of technology corresponding to a product innovation which is then followed by subsequent process innovations. This idea can be traced back to the pioneering work by Abernathy and Utterback (1975, 1978). Lambertini and Mantovani (2009) consider a multiproduct monopolist that simultaneously pursues process and product innovations. They show that the path to the saddle-point steady state involves some substitutability between the two innovation types.
} 
where $\delta>0$ is the constant rate of efficiency reduction due to the ageing of technology. ${ }^{9}$ The cost of R\&D efforts per unit of time is given by:

$$
\Gamma(k(t))=b k(t)^{2},
$$

where $b>0$ is inversely related to the cost-efficiency of the R\&D process. Hence, the $\mathrm{R} \& \mathrm{D}$ process exhibits decreasing returns to scale. ${ }^{10}$ The monopolist discounts the future with the constant rate $\rho>0$.

Instantaneous profit is:

$$
\pi(q, k, c)=(A-q-c) q-b k^{2},
$$

yielding total discounted profit:

$$
\Pi(q, k, c)=\int_{0}^{\infty} \pi(q, k, c) e^{-\rho t} d t .
$$

The optimal control problem for the monopolist is to find controls $q^{*}$ and $k^{*}$ that maximize the profit functional $\Pi$ subject to the state equation (2), the initial condition $c(0)=c_{0}$, and two boundary conditions which must hold at all times: $q \geq 0$ and $k \geq 0$. Note that according to (2), if $c_{0}>0$, then $c(t)>0$ for all $t$. The set of all possible states at each time $t$ is given by $c \in[0, \infty)$.

The model has four parameters: $A, b, \delta$, and $\rho$. The analysis can be simplified by considering a rescaled version of the model which, as defined in Lemma 1, carries only two parameters: $\phi$ and $\tilde{\rho}$. That is, rescaling the model translates the four-dimensional parameter space into a two dimensional one.

Lemma 1. By choosing the units of $t, q, c$, and $k$ appropriately, we can assume $A=1$,

\footnotetext{
${ }^{9}$ In specifying a positive, exogenous depreciation rate, we follow the literature (e.g., Lambertini and Mantovani (2009)). There are good reasons to assume an exogenous increase in marginal costs over time. Production costs include complementary inputs that are typically bought. The nominal price of these complementary inputs might increase over time. Also, incorporating them into the production process becomes ever more costly due to their inherent evolution over time (e.g., installing a newer version of software on obsolete hardware can significantly slow down performance). Further, maintenance costs can increase over time and there is always a potential that some inputs will become obsolete (e.g., with the invention of computers, the typewriter became antiquated). Alternatively, $\delta$ is considered to represent increasing opportunity costs over time. Note that the equilibria for a non-positive depreciation rate follow trivially. For $\delta$ is zero, consider $\delta$ very close to zero. Such small depreciation rate pushes the level of the initial technology that will not be developed further far beyond the choke price as only minuscule investments are needed to reduce marginal costs over time. Indeed, for negative $\delta$, every initial technology will be developed further as there is an exogenous reduction in marginal costs over time, yielding a steady state with no production costs.

${ }^{10}$ Again, we follow the literature with this assumption (see, e.g., d'Aspremont and Jacquemin (1988), Kamien et al. (1992), or Qiu (1997)). The evidence is mixed however. Schwartzman (1976) for instance documents significant economies of scale in R\&D. But Madsen (2007) is unable to reject the hypothesis of constant returns to scale in the number of patents applied for. Moreover, for the pharmaceutical industry, Vernon and Gusen (1974) and Graves and Langowitz (1993) concludes that firms experience decreasing returns to scale in R\&D. And Adams and Griliches (1996) conclude that there are strong diseconomies of scale in the production of scientific articles.
} 
$b=1$, and $\delta=1$. This yields the following, rescaled model:

$$
\begin{gathered}
\tilde{\pi}(\tilde{q}, \tilde{k}, \tilde{c})=(1-\tilde{q}-\tilde{c}) \tilde{q}-\tilde{k}^{2}, \\
\tilde{\Pi}(\tilde{q}, \tilde{k}, \tilde{c})=\int_{0}^{\infty} \tilde{\pi}(\tilde{q}, \tilde{k}, \tilde{c}) e^{-\tilde{\rho} \tilde{t}} d \tilde{t} \\
\dot{\tilde{c}}=\tilde{c}(1-\phi \tilde{k}), \quad \tilde{c}(0)=\tilde{c}_{0}, \quad \tilde{c} \in[0, \infty) \forall \tilde{t} \in[0, \infty) \\
\tilde{q} \geq 0, \quad \tilde{k} \geq 0 \\
\tilde{\rho}>0, \quad \phi>0
\end{gathered}
$$

with conversion rules: $q=A \tilde{q}, c=A \tilde{c}, k=\frac{A}{\sqrt{b}} \tilde{k}, t=\frac{\tilde{t}}{\delta}, \pi=A^{2} \tilde{\pi}, \phi=\frac{A}{\delta \sqrt{b}}, \rho=\tilde{\rho} \delta$.

Proof. See Appendix A.

The rescaled model introduces a new parameter $\phi=A /(\delta \sqrt{b})$, which captures the efficiency of the R\&D process: a higher $b$ means that each unit of R\&D effort costs the firm more, whereas a higher $\delta$ implies that each unit of R\&D effort reduces the marginal cost by less. Therefore, a higher (lower) $\phi$ corresponds to a more (less) efficient $R \& D$ process. For notational convenience, we henceforth omit tildes.

To solve the dynamic optimization problem, we introduce the current-value Pontryagin function ${ }^{11}$

$$
P(c, q, k, \lambda)=(1-q-c) q-k^{2}+\lambda c(1-\phi k),
$$

where $\lambda$ is the current-value co-state variable. It measures the marginal worth of the increment in the state $c$ at time $t$ when moving along the optimal path. As the marginal cost is a "bad", we expect $\lambda(t) \leq 0$ along optimal trajectories.

Pontryagin's Maximum Principle states that if the pair $\left(c^{*}, q^{*}, k^{*}\right)$ is an optimal solution, then there exists a function $\lambda(t)$ such that $c^{*}, q^{*}, k^{*}$, and $\lambda$ satisfy the following conditions:

1.) $q^{*}$ and $k^{*}$ maximize the function $P$ for each $t$ :

$$
P\left(c^{*}, q^{*}, k^{*}, \lambda\right)=\max _{(q, k) \in \mathbb{R}_{+}^{2}} P\left(c^{*}, q, k, \lambda\right),
$$

2.) $\lambda$ is a solution to the following co-state equation:

$$
-\frac{\partial P}{\partial c}=\dot{\lambda}-\rho \lambda \quad \Leftrightarrow \quad \dot{\lambda}=q+(\rho-1+\phi k) \lambda,
$$

\footnotetext{
${ }^{11}$ Also called pre-Hamilton or un-maximized Hamilton function.
} 
which is evaluated along with the equation for the marginal cost

$$
\dot{c}=c(1-\phi k)
$$

and the initial condition $c(0)=c_{0}$.

Let $q=Q(c, \lambda)$ and $k=K(c, \lambda)$ solve the problem $\max _{(q, k)} P(c, q, k, \lambda)$ for every $(c, \lambda)$. We define the current-value Hamilton function

$$
H(c, \lambda)=P(c, Q(c, \lambda), K(c, \lambda), \lambda) .
$$

The above necessary conditions (12)-(13) for an optimal solution are complemented by two transversality conditions: ${ }^{12}$

$$
\lim _{t \rightarrow \infty} e^{-\rho t} H(c, \lambda)=0
$$

and

$$
\lim _{t \rightarrow \infty} e^{-\rho t} \lambda c=0
$$

If problem (12) has a solution, it necessarily satisfies the following Karush-KuhnTucker conditions:

$$
\begin{array}{ll}
\frac{\partial P}{\partial q}=1-2 q-c \leq 0, & q \frac{\partial P}{\partial q}=0, \\
\frac{\partial P}{\partial k}=-2 k-\phi \lambda c \leq 0, & k \frac{\partial P}{\partial k}=0 .
\end{array}
$$

Conditions (18) imply that either i) $q=0$ and $c \geq 1$ or $i i) q>0$ and $c<1 .^{13}$ In particular:

$$
q^{*}=\left\{\begin{array}{cll}
(1-c) / 2 & \text { if } & c<1 \\
0 & \text { if } & c \geq 1 .
\end{array}\right.
$$

Conditions (19) imply that either $i) k=0$ and $\frac{\partial P}{\partial k} \leq 0$ (implying $\lambda \geq 0$ ) or $\left.i i\right) k>0$ and $\frac{\partial P}{\partial k}=0$. In particular:

$$
k^{*}=\left\{\begin{array}{ccc}
-\frac{\phi}{2} \lambda c & \text { if } & \lambda \leq 0, \\
0 & \text { if } & \lambda>0 .
\end{array}\right.
$$

The above conditions for optimal production yields two regimes of the state-control system. The first is characterized by positive production, in the second there is no production.

\footnotetext{
${ }^{12}$ The necessity of (16), which allows exclusion of non-optimal trajectories, was proven by Michel (1982) Kamihigashi (2001) proves the necessity of (17).

${ }^{13}$ Observe that in the non-rescaled model, the analogous condition for positive production is that $c(t)<A$.
} 


\subsection{The system with positive production}

In the region where $c<1$, using (13), (14), (20), and (21), we obtain the following optimal state and co-state dynamics:

$$
\left\{\begin{array}{l}
\dot{c}=c\left(1+\frac{1}{2} \phi^{2} \lambda c\right) \\
\dot{\lambda}=\frac{1}{2}(1-c)+\left(\rho-1-\frac{1}{2} \phi^{2} \lambda c\right) \lambda
\end{array}\right.
$$

where $\lambda \leq 0 .{ }^{14}$ Equation (21) depends on the co-state and state variable. Note that the correspondence between $k$ and $\lambda$ is one-to-one if, and only if, $\lambda \leq 0$, and that therefore the system can be re-written in the state-control form.

Differentiate equation (21) with respect to time to obtain the dynamic equation

$$
\frac{d k}{d t} \equiv \dot{k}=-\frac{\phi}{2}(\dot{\lambda} c+\lambda \dot{c})
$$

Use (13) and (14) for $\dot{\lambda}$ and $\dot{c}$ respectively. Note that equation (21) also implies that

$$
\lambda=-\frac{2 k}{\phi c}
$$

Substitute this expression into (23), together with the expression for the optimal output level $(q=(1-c) / 2)$, to obtain:

$$
\dot{k}=\rho k-\frac{\phi}{4} c(1-c)
$$

Hence, the system with positive production $(c<1)$ in the state-control form consists of the following two differential equations:

$$
\left\{\begin{array}{l}
\dot{k}=\rho k-\frac{\phi}{4} c(1-c), \\
\dot{c}=c(1-\phi k) .
\end{array}\right.
$$

\subsection{The system with zero production}

In the region $c \geq 1$, substituting (13), (14), and (24) into (23) and imposing $q=0$, gives the following expression:

$$
\dot{k}=\rho k \text {. }
$$

Hence, the state-control system with zero production consists of the following two differential equations:

$$
\left\{\begin{array}{l}
\dot{k}=\rho k, \\
\dot{c}=c(1-\phi k) .
\end{array}\right.
$$

\footnotetext{
${ }^{14}$ The differential equations in the text are all valid for $\lambda \leq 0$. For a complete specification of optimal state and co-state dynamics (including the case when $\lambda>0$ ), we refer the reader to Appendix F.
} 
The state-co-state analogue is:

$$
\left\{\begin{array}{l}
\dot{c}=c\left(1+\frac{1}{2} \phi^{2} \lambda c\right), \\
\dot{\lambda}=\left(\rho-1-\frac{1}{2} \phi^{2} \lambda c\right) \lambda .
\end{array}\right.
$$

The Hamilton function, obtained by substituting (21) and appropriate expressions for $q$ from (20) into the Pontryagin function (11), is given by

$$
H(c, \lambda)=\left\{\begin{array}{ccc}
\frac{1}{4}(1-c)^{2}+\frac{1}{4} \phi^{2} \lambda^{2} c^{2}+\lambda c & \text { if } & c \in[0,1), \\
\frac{1}{4} \phi^{2} \lambda^{2} c^{2}+\lambda c & \text { if } & c \in[1, \infty),
\end{array}\right.
$$

where $\lambda \leq 0$. The state-control analogue is then

$$
H(c, k)=\left\{\begin{array}{ccc}
\frac{1}{4}(1-c)^{2}+k\left(k-\frac{2}{\phi}\right) & \text { if } & c \in[0,1), \\
k\left(k-\frac{2}{\phi}\right) & \text { if } & c \in[1, \infty),
\end{array}\right.
$$

where $k \geq 0$.

\section{Steady-state solutions}

The steady-state solutions of (26) and (28) are obtained by imposing the stationarity conditions $\dot{k}=0$ and $\dot{c}=0$.

Lemma 2. The "no-production" state-control system (28) has no steady state in the region where it is defined.

Proof. See Appendix B.

Consider now system (26). Imposing the stationarity condition $\dot{k}=0$, we obtain

$$
k^{M}=\frac{\phi}{4 \rho} c(1-c) \geq 0, \quad \forall c \in[0,1],
$$

where superscript $M$ stands for Monopoly. ${ }^{15}$ Steady-state marginal cost follows from inserting (32) into the state dynamics (14) and imposing the stationarity condition $\dot{c}=0$ :

$$
\dot{c}=c\left(1-\frac{\phi^{2}}{4 \rho} c(1-c)\right)=0 .
$$

This yields:

$$
c^{M}=0, \quad c^{M}=\frac{1}{2} \pm V,
$$

\footnotetext{
${ }^{15}$ All steady-state values have superscript $M$.
} 
where

$$
V=\frac{1}{2} \sqrt{1-\frac{16 \rho}{\phi^{2}}} .
$$

Observe that $V$ is real if, and only if, $\phi \geq 4 \sqrt{\rho}$, in which case $V \in\left[0, \frac{1}{2}\right)$ and $c^{M} \in$ $[0,1)$. The solution to the system is summarized in Proposition 1 and is depicted in Figure 1.

Proposition 1. If $\phi>4 \sqrt{\rho}$, the state-control system with positive production (26) has three steady states:

i) $\left(c^{M}, k^{M}\right)=(0,0)$ is an unstable node,

ii) $\left(c^{M}, k^{M}\right)=\left(\frac{1}{2}+V, \frac{1}{\phi}\right)$ is either an unstable node or an unstable focus, and

iii) $\left(c^{M}, k^{M}\right)=\left(\frac{1}{2}-V, \frac{1}{\phi}\right)$ is a saddle-point steady state.

At $\phi=4 \sqrt{\rho}$, a so-called "saddle-node bifurcation" occurs as the last two steady states coincide and form a so-called "semi-stable" steady state.

If $\phi<4 \sqrt{\rho}$, the state-control system with positive production has one single steady state: the origin $\left(c^{M}, k^{M}\right)=(0,0)$, which is unstable.

Proof. See Appendix C.

In the saddle-point steady state, instantaneous marginal cost, output, and profits are, respectively:

$$
\begin{gathered}
c^{M}=\frac{1}{2}-V, \\
q^{M}=\frac{1}{4}+\frac{V}{2}, \\
\pi^{M}=\frac{1}{16}(1+2 V)^{2}-\frac{1}{\phi^{2}} .
\end{gathered}
$$

\subsection{Comparative statics}

The relevant comparative statics are as follows (for a graphical illustration of these relations, see Figure 2): ${ }^{16}$

Lemma 3. Steady-state marginal cost is increasing in $b, \delta$, and $\rho$, and decreasing in $\phi$; steady-state quantity, profit, consumers' surplus, and total surplus are decreasing in $b, \delta$, and $\rho$, and increasing in $\phi$.

\footnotetext{
${ }^{16}$ The graphs are indicative only. They are drawn for the admissible range of parameters in the sense that for these values the saddle-point steady state exists. However, and as discussed further below, the steady-state will not be reached for all these parameter values.
} 


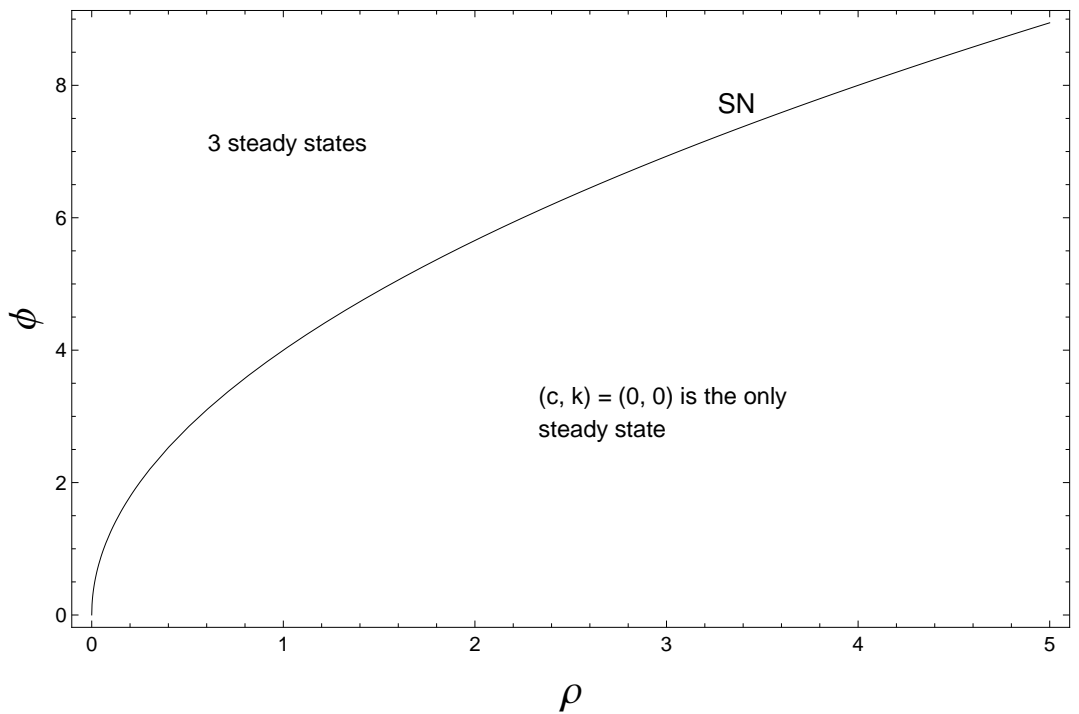

Figure 1: Steady states of the state-control system. The the saddle-node bifurcation (SN) curve is defined by $\phi=4 \sqrt{\rho}$. Passing through it from above, the two steady states other than origin coincide, form a "semi-stable" steady state, and then disappear, leaving one steady state to exist only.

\section{Proof. See Appendix D.}

Recall that $\delta$ accounts for an exogenous decrease in production efficiency due to the ageing of technology. The monopolist does not need to exert the desired level of the R\&D effort at once but can smooth it over infinitely many instants. With a convex $R \& D$ cost function this enables him to reduce his investment costs. The higher the rate at which the technology depreciates, the less advantageous this smoothing will be, as a larger part of what the monopolist has invested is lost in every next instant. Further, in the steady state, the monopolist invests just enough to keep his marginal cost constant at the desired level. Hence, the higher is $\delta$, the more costly this maintenance will be, as a larger part of the technology must be replaced in every period.

The higher is $b$, the more it costs a firm to implement a particular R\&D effort in every instant. As an increase in either $\delta$ or $b$ makes implementing every desired R\&D effort level more costly, an increase (decrease) in $b$ and/or $\delta$ increases (decreases) the steady-state level of marginal cost and decreases (increases) the steady-state output, profits and total surplus, as stated in Lemma 3 above. ${ }^{17}$

\footnotetext{
${ }^{17}$ Steady-state marginal cost in the non-rescaled model equals $c^{M}=\left(A-\sqrt{A^{2}-16 b \delta \rho}\right) / 2$. For the steady-state values of other variables of the non-rescaled model, see Appendix D.
} 


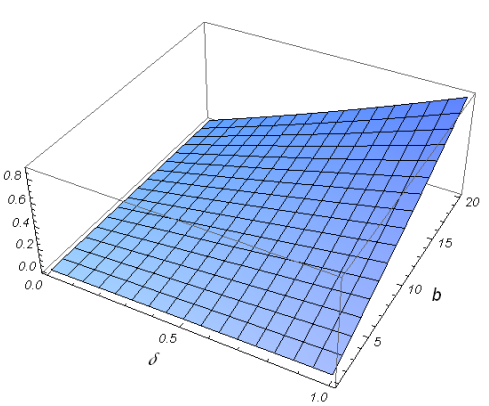

(a) marginal cost

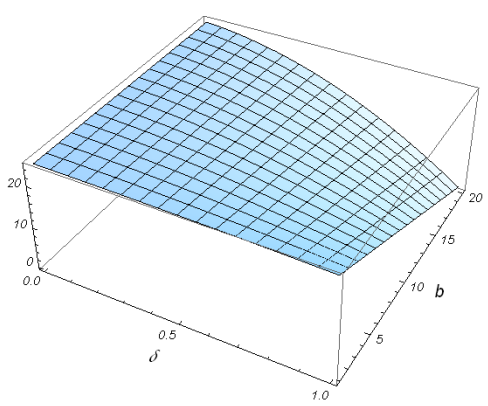

(c) profit

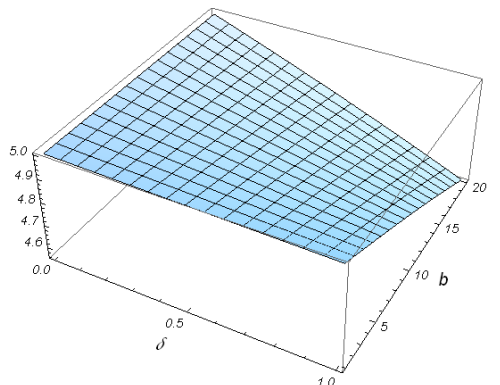

(b) quantity

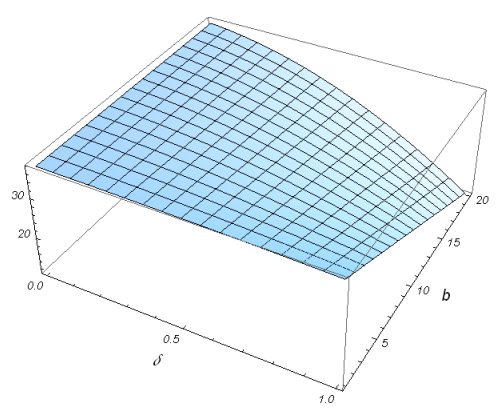

(d) total surplus

Figure 2: Steady-state instantaneous values of the marginal cost, quantity, profit, and total surplus. Graphs are drawn for $A=10$ and $\rho=0.1$. Variables and parameters refer to the non-rescaled model.

\section{Global optimality and industry dynamics (bifurcations of optimal vector fields)}

Thus far we have focused on the steady state. Obviously, the stable manifold of the saddle-point steady state is one of the candidates for an optimal solution. However, as the following remark shows, it may not (always) be the true optimum.

Remark 1. The Pontryagin function of the optimization problem as given in (11) is not jointly concave in the state and control variables. Hence, necessary conditions for an optimum are not necessarily sufficient.

Proof. See Appendix E.

Therefore, we have to carry out a detailed bifurcation analysis to assess the dependence of the solution structure on the model parameters. ${ }^{18} 19$ This yields a com-

\footnotetext{
${ }^{18}$ For a recent discussion of the use of bifurcation theory in analyzing non-convex optimal control problems with multiple equilibria, see Kiseleva and Wagener (2010).

${ }^{19}$ Note that Remark 1 applies to all related papers as well, including Lambertini and Mantovani (2009), Cellini and Lambertini (2009), and Kováč et al. (2010). Without further analysis it remains unclear whether these papers discuss truly optimal behavior. A sufficiency proof that does not rely on joint concavity of state and control variables can be given by adapting the Stalford sufficiency theorem (Stalford, 1971) to an infinite horizon setting, as for instance in Polasky et al. (2011).
} 
plete overview of how optimal R\&D investment and concomitant production respond to changes in the initial level of marginal cost and to varying characteristics of the R\&D process. To analyze global optimality, we have to inspect the complete state-control space, which is the set of solutions $(c(t), k(t))$ of the state-control system considered as parameterized curves (trajectories) in a plane. Its general form is sketched in Figure 3 .

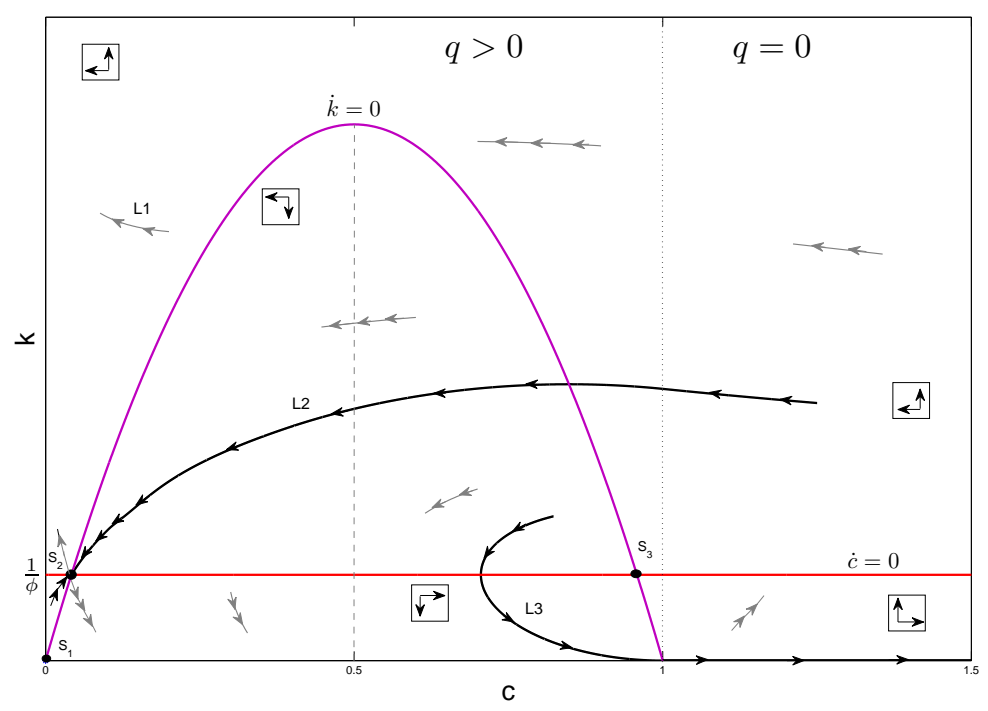

Figure 3: Illustrative sketch of the state-control space.

The dotted vertical line $c=1$ separates the region with zero production from the region with a positive level of production. In the left region, the parabola which achieves its maximum at $c=1 / 2$ is the locus $\dot{k}=0$. The loci representing points where $\dot{c}=0$ are the horizontal line $k=1 / \phi$ and the vertical line $c=0$ which coincides with the $k$-axis. Loci $\dot{c}=0$ and $\dot{k}=0$ intersect in the saddle-point steady state $\left(S_{2}\right)$ and the two unstable steady states $\left(S_{1}\right.$ and $\left.S_{3}\right)$. The black arrows in squares indicate the direction of trajectories in each respective region. The horizontal arrows indicate an increase or a decrease in $c$, whereas the vertical ones refer to changes in $k$. A number of trajectories is indicated by grey arrows. Together, the trajectories cover the entire space. However, there are only two trajectories leading to the saddle-point steady state; these are called "stable paths" or "stable manifolds". They are indicated by the connected black arrows pointing towards $S_{2}$. The lighter arrows pointing away from $S_{2}$ indicate the two unstable paths. Trajectories spiral out from $S_{3}$ in a counter-clockwise motion.

The solution to the state-control system (28), which yields trajectories in the region 
with zero production, is given by:

$$
\begin{aligned}
& k(t)=C_{1} e^{\rho t}, \\
& c(t)=C_{2} e^{\left(t-\frac{e^{\rho t} \phi C_{1}}{\rho}\right)},
\end{aligned}
$$

where $C_{1}$ and $C_{2}$ are positive real constants.

A solution to the state-control system (26), which yields trajectories in the region with positive production, cannot be obtained analytically; we therefore make use of geometrical-numerical techniques. ${ }^{20}$

A particular trajectory is a candidate for an optimal solution if it satisfies all necessary conditions: those given by Pontryagin's Maximum Principle together with transversality conditions (16) and (17). As Figure 3 shows, there exists a whole range of solutions $(c(t), k(t))$ to the state-control system. These trajectories all satisfy the conditions of Pontryagin's Maximum Principle. By ruling out those trajectories that do not satisfy the transversality conditions, we are left with the candidates for an optimal solution.

Lemma 4. The points in Figure 3 in the set

$$
\{(c, k): c \in(0,1), k=0\}
$$

cannot be a part of any optimal trajectory.

Proof. See Appendix F.

Lemma 5. All trajectories along which $k \rightarrow \infty$ and $c \rightarrow 0$ as $t \rightarrow \infty$ can be ruled out as optimal solutions.

Proof. See Appendix G.

For instance, the trajectory denoted by $L_{1}$ in Figure 3 is in the set of Lemma 5.

Lemma 6. The trajectory through the point $(c, k)=(1,0)$ satisfies the transversality conditions (16) and (17).

Proof. See Appendix H.

In Figure 3, the trajectory of Lemma 6 is labeled $L_{3}$. We call this the "exit trajectory" as it implies that the firm (eventually) exits the market. Indeed, at some stage the exit trajectory enters the region where $c \geq 1$, at which point both R\&D investment and production come to a halt. ${ }^{21}$

\footnotetext{
${ }^{20}$ See also Judd (1998) and Wagener (2003). All numerical simulations were done in Matlab.

${ }^{21}$ Strictly speaking, the firm's marginal cost continue to increase along the exit trajectory due to the positive depreciation rate $\delta$. However, we interpret any situation in which the firm stays inactive as if it has left the market.
} 
Notice that the stable path (denoted by $L_{2}$ in Figure 3) to the saddle-point steady state $\left(S_{2}\right)$ also satisfies the transversality conditions. Notably, along this trajectory, both $c$ and $k$ converge to finite limits and, therefore, so does the co-state variable (24).

As the following corollary to the above lemmas shows, we are left with two candidates for an optimal solution.

Corollary 1. The set of candidates for an optimal solution consist of the stable path of the saddle-point steady state and the trajectory through the point $(c, k)=(1,0)$.

Proof. See Appendix I.

To find an optimal solution from the candidates, we make use of the following lemma.

Lemma 7. If the pair $(c(\cdot), q(\cdot), k(\cdot))$ satisfies the necessary conditions (12)-(17), then

$$
\begin{aligned}
\Pi(c, q, k) & =\int_{0}^{\infty} \pi(q, k, c) e^{-\rho t} d t \\
& =\frac{1}{\rho} P(c(0), q(0), k(0), \lambda(0)) \\
& =\frac{1}{\rho} H(c(0), k(0)),
\end{aligned}
$$

where $H$ is the Hamilton function in state-control form.

Proof. See, e.g., Grass et al. (2008), ch. 3, pp. 161-162.

Lemma 7 is useful whenever several paths satisfy all necessary conditions for an optimal solution and we have to determine which one yields the highest discounted profit flow. According to Lemma 7, these flows are equal to the rescaled values of the Hamiltonian (31) at the corresponding initial points.

Figure 4 provides an example in which the optimal solution is the stable path leading to the saddle-point steady state. Note that the initial marginal costs can be higher than $c=1$. In that case, the optimal decision leads the firm to first produce nothing but to invest increasingly in the reduction of its marginal costs. When marginal costs are below one, it starts producing. The level of R\&D investments then gradually decreases to its long-run steady-state level. This is exactly the scenario behind the story of cellophane. ${ }^{22}$

Note that having a phase of zero production as a part of the stable path is possible only if the range of initial marginal costs is not bounded to be below the choke price. In this phase instantaneous profits are negative, but total discounted profits are positive;

\footnotetext{
${ }^{22}$ Another example is the petrochemical industry. Stobaugh (1988) finds that R\&D efforts aimed at process innovations increase every year in the period following the initial product innovation, only to decrease again after that. He also documents that the probability of the next process innovation being major is decreasing over time.
} 


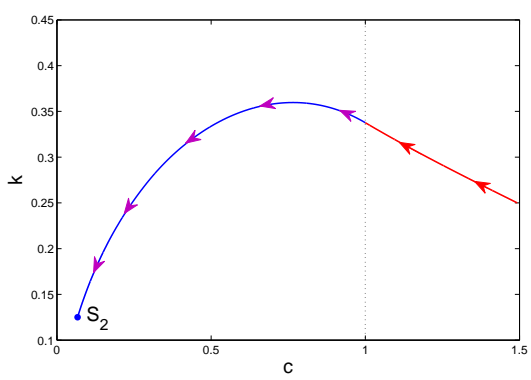

(a)

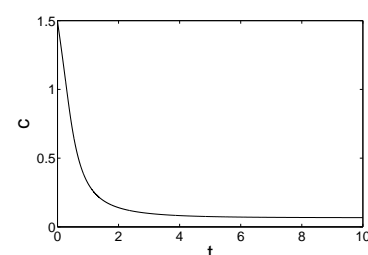

(c)

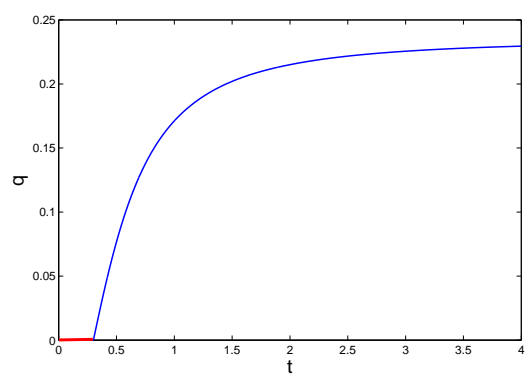

(b)

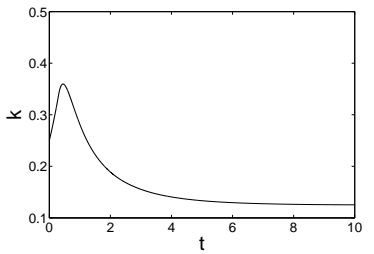

(d)

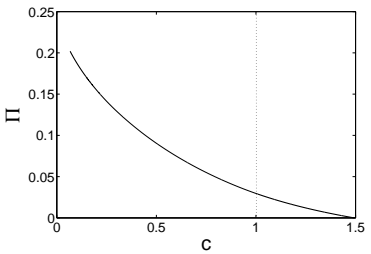

(e)

Figure 4: State-control space (a), time paths for quantity (b), marginal cost (c), and $R \& D$ effort $(d)$, and the correlation diagram between the total discounted profit and marginal cost $(e)$, respectively. All plots show curves for parameter values $(\rho, \phi)=$ $(1,8)$.

the monopolist invest in $\mathrm{R} \& \mathrm{D}$ because once marginal costs are below the choke price, production becomes profitable. ${ }^{23}$

In the example of Figure 4, the monopolist optimally selects an appropriate $k$ such that $\left(c_{0}, k_{0}\right)$ is located on the stable path. However, as the Arrow-Mangasarian sufficiency conditions are not satisfied (see Remark 1), an investment policy that follows the stable path is not necessarily optimal at all times. Indeed, it could not even be a possible solution as the saddle-point steady state does not exist for all parameter values (recall Proposition 1). That is, the solution structure depends on the system's parameters.

Figure 5 presents the bifurcation diagram, which shows the optimal R\&D investments for varying parameter values. ${ }^{24}$ Every combination of the exogenous parameters yields a point on this diagram. It consists of five distinct regions, each representing a particular structure of the set of optimal solutions. In general, either there exists an upper bound of initial marginal cost below which the monopolist selects the stable path towards the saddle-point steady state, or the (new) technology is always phased out by a

\footnotetext{
${ }^{23}$ Related, the literature on optimal technology adoption predicts a substantial time lag between the discovery of a new technology and its adoption (see, e.g., Hoppe (2002) for an overview). Delaying the adoption provides additional information as to the profitability of a new technology; it is also rational if a better technology is expected to become available in the near future (see also Doraszelski (2004)). In this literature, however, technological progress is exogenous.

${ }^{24}$ The bifurcation diagram in Figure 5 is drawn for the parameters of the non-rescaled model.
} 


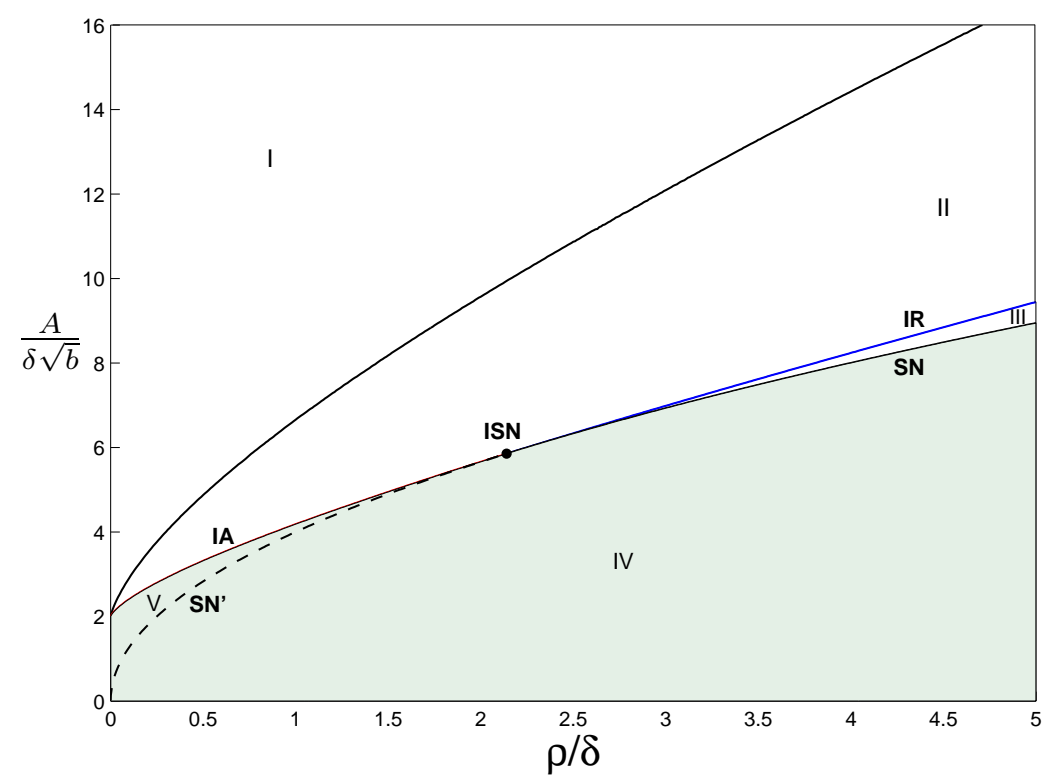

Figure 5: Bifurcation diagram. The uppermost curve represents parameter values for which the indifference point between the stable path and the exit trajectory is exactly at $c=1$. SN stands for the "saddle-node bifurcation curve", SN' for the "inessential saddle-node bifurcation curve", IA for the "indifference-attractor bifurcation curve", and IR for the "indifference-repeller bifurcation curve". ISN indicates the "indifference-saddle-node point", which is the point from which indifference-attractor, indifference-repeller, saddle-node and inessential saddle-node curves emanate. Its coordinates are $\tilde{\rho} \approx 2.14$ and $\phi \approx 5.85$. Roman numerals (I-V) indicate the corresponding parameter regions discussed in the main text. The shaded region (regions IV and V) indicates the parameter region for which the firm always exits the market. Notice that the axes are excluded from the admissible parameter space. The curve representing indifference points at $c=1$ obtains a value of $\phi \approx 2.00$ for $\tilde{\rho}=1 \times 10^{-5}$.

firm (eventually) exiting the market. Hence, if it exists, convergence to the saddle-point steady state along the stable path is locally optimal only; it is never optimal globally.

Solutions to the optimization problem for parameters outside the shaded region in Figure 5 are characterized by the presence of threshold values; the stable path of the saddle-point steady state is optimal if the initial marginal cost is below some threshold level, otherwise the exit trajectory is optimal. The threshold point can be either an indifference point ${ }^{25}$ or a repeller. Indifference points are initial states $c=c_{0}$ for which the firm is indifferent between several possible investment policies (i.e., the stable path and the exit trajectory). A repeller differs from an indifference point in that at a repelling point, it is optimal to stay at that point forever after. ${ }^{26}$

\footnotetext{
${ }^{25}$ Also called Skiba points or DNSS points (see Grass et al. (2008)).

${ }^{26}$ More formally, a threshold point separates two basins of attraction: the initial values of the marginal cost below these points constitute the basin of attraction of the saddle-point steady-state; the initial values
} 

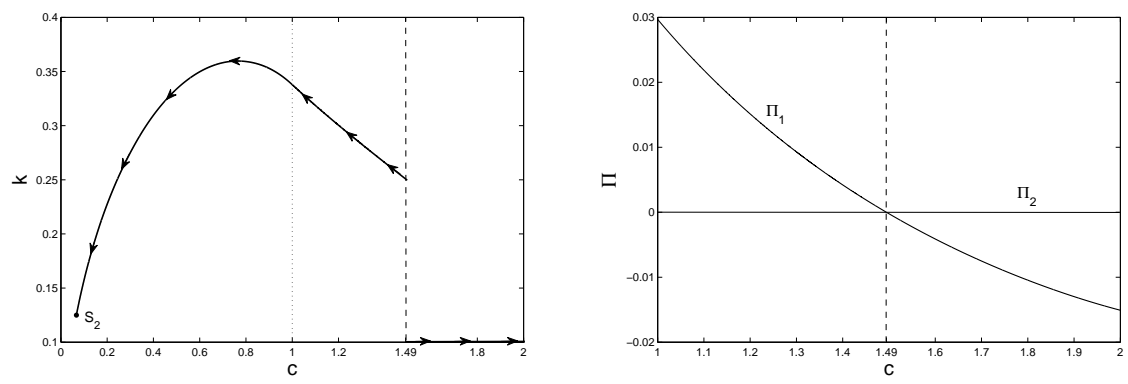

Figure 6: State-control space and the total profit function when the indifference point is in the region with zero production. The plots are drawn for parameter values $(\rho, \phi)=$ $(1,8)$.

\subsection{Region I}

In Region I, the point of indifference is above one. Therefore, the system will converge to the saddle-point steady state for all initial technologies $c_{0} \in(0,1]{ }^{27}$ A typical example is depicted in Figure 6. The arrows indicate the direction of motion along optimal trajectories. The two regions (production, no production) are split by the dotted vertical line $c=1$. For a given $\rho$, Region $I$ is characterized by a relatively high $\phi$, that is, by relatively large demand and/or high R\&D efficiency. In such a favorable environment, the monopolist can compensate for his early stage losses if, initially, production is postponed as that would yield negative mark-ups. There exists, however, a finite upper bound $\hat{c}>1$ for the initial marginal costs $c_{0}$ beyond which future profits are not enough to compensate for short-run losses ( $\hat{c} \approx 1.49$ in the case considered). In that case, the initial technology will not be developed further. In Figure 6, the point of indifference (indicated by a dashed vertical line) occurs where the total profit function for the stable path $\left(\Pi_{1}\right)$ in the right-hand picture obtains a value of zero (which is the value of the total profit function for the exit trajectory $\Pi_{2}$ in the region $c \geq 1$ ). As the total profit beyond this point is negative (the firm makes a net loss), the firm prefers not to invest at all. Starting from any initial value of marginal cost below the indifference point, the firm's optimal decision is to follow the stable path. The exact position of the indifference point is defined in Lemma 8.

Lemma 8. If an indifference point between the stable path and the exit trajectory does exist in the region with zero production $(c \geq 1)$, it is given by the value of the marginal cost $\hat{c} \geq 1$ for which the point $(c, k)=\left(\hat{c}, \frac{2}{\phi}\right)$ lies on the stable path. For $c_{0}=\hat{c}$, total discounted profits are zero for both trajectories.

of the marginal cost above these points constitute the basin of attraction to exit the market. An indifference point lies in both basins of attractions, a repeller in neither.

${ }^{27}$ Note that $c_{0}=0$ corresponds to an unstable steady state; for this trivial value of the marginal cost, the monopolist stays at that point forever. 

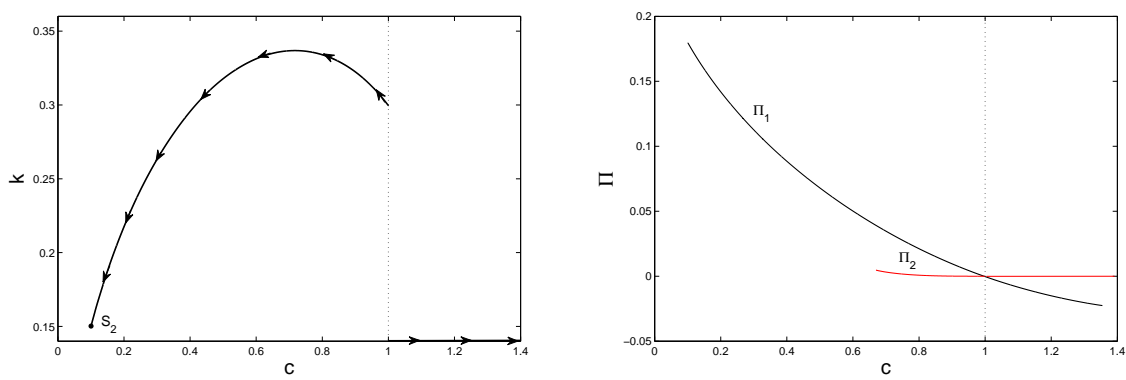

Figure 7: State-control space and the total profit functions when the indifference point is exactly on the boundary between the two regions $(\hat{c}=1)$. The plots are drawn for parameter values $(\rho, \phi)=(1,6.655)$.

Proof. See Appendix J.

Region $I$ is bounded from below by the curve for which the point of indifference is exactly at $c=1$. This particular situation is depicted in Figure 7: for all initial values of the marginal cost $c_{0} \in(0,1)$, the optimal-solution trajectory is the stable path of the saddle-point steady state, while for $c_{0} \in(1, \infty)$, it is optimal not to produce and invest anything; at $c_{0}=\hat{c}=1$, either strategy is optimal. The right-hand picture shows that the total profit $\Pi_{1}$ for the stable path is zero at exactly $c=1$. For comparison, the total profit $\Pi_{2}$ on the exit trajectory, which passes through the point $(c, k)=(1,0)$, is plotted up to its conjugate point (the last point on the trajectory where marginal cost is still decreasing; see also footnote 30 below).

The stable path in the region with zero production $(c \geq 1)$ is characterized by a decreasing $k$ and an increasing $c$ as $t \rightarrow-\infty$ (see (39)-(40) and Figure 6). From Lemma 8 follows that the indifference point between the stable path and the exit trajectory is exactly at $c=1$ when the stable path reaches $k=2 / \phi$ exactly at $c=1$ (see Figure 7). As investment is increasing in $\phi$ (for a given $\rho$ ), we can find for every $\rho$ a high enough $\phi$ such that the indifference point is either at $c=1$ or in the region with $c \geq 1$. A higher $\phi$ for a given $\rho$ in Region I just moves the indifference point further beyond $c=1$. Consequently, Region $I$ is not bounded from the right. Moreover, as $k$ decreases along the stable path as $t \rightarrow-\infty$, at some instant the stable path, having a value of $k$ at $c=1$ above $k=2 / \phi$, reaches the point with $k=2 / \phi$ for some $c>1$ as $t \rightarrow-\infty$. Therefore, for parameters in Region I, we always have an indifference point at some $c=\hat{c} \geq 1$ and, in particular, the convergence to the saddle-point steady-state is for these parameters guaranteed if $c_{0} \in(0,1]$, but not for all $c_{0} \in(1, \infty)$.

Figure 8 illustrates how the indifference point varies with the parameters. ${ }^{28}$ Clearly, for a given discount rate $\rho$, the more efficient is the R\&D process, the larger will be

\footnotetext{
${ }^{28}$ Figure 8 is drawn for the parameters of the non-rescaled model.
} 


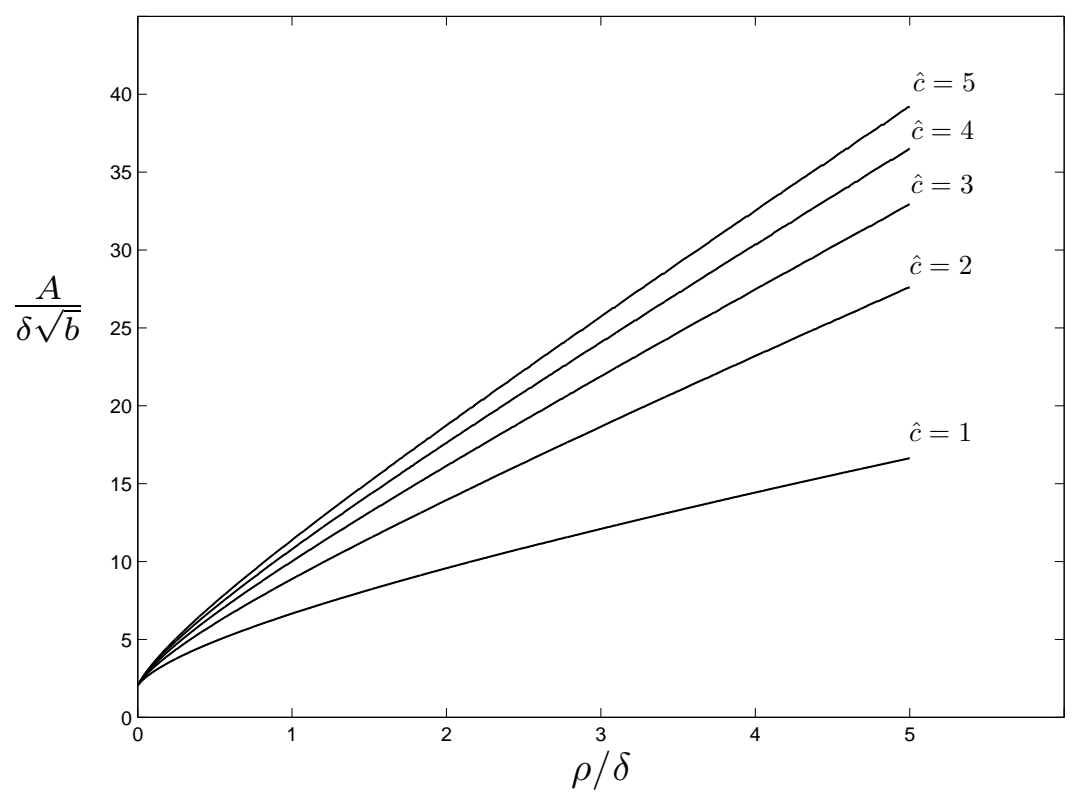

Figure 8: Dependence of the indifference point $\hat{c}$ on the model parameters. Curves are drawn for five fixed values of $\hat{c}$. Given $\rho$, a higher value of $A$ or a lower value of $b$ and/or $\delta$ increases the value of marginal cost at the indifference point, and vice versa. This effect is larger, the smaller the value of $\rho$. An increase in $\rho$ reduces the critical value of marginal cost, ceteris paribus.

the range of initial marginal costs that lead the monopolist to select the stable path. Likewise, a higher choke price corresponds to a higher value of initial marginal cost at the indifference point. This has an important implication: market characteristics that influence future production, and hence profitability, affect whether or not a monopolist starts investing in developing a new technology. That is, if market regulations are such that future mark-ups are reduced, for instance because the monopolist is forced to price closer to its marginal costs, the range of initial technology levels that repay development shrinks. The loss of total surplus this reduction brings about is a hidden cost of competition policies if the indifference point is above $c=1$, as in that case there is initially no production. Not pursuing the development of the technology does consequently not surface as an immediate reduction in total surplus. Yet, these indirect costs should be taken into account when assessing properly the trade-off between static and dynamic efficiency.

\subsection{Regions II and III}

In Region II, the indifference point $\hat{c}$ is below $c=1$. This implies in particular that at the indifference point the monopolist produces a positive quantity. Figure 9 illustrates 

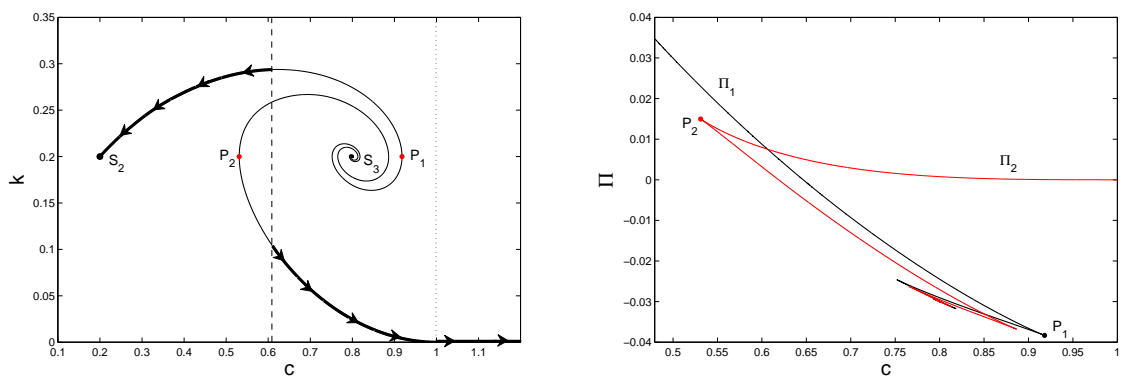

Figure 9: State-control space and the total profit functions when the indifference point is within the region with positive production. The plots are drawn for parameter values $(\rho, \phi)=(1,5)$. The optimal-solution trajectories are marked by thick lines. The two candidates for the optimal solution, which both spiral out of the unstable steady state $S_{3}$, are the stable path and the exit trajectory.

this case. The indifference point occurs where the two respective total profit functions intersect, as indicated in the right-hand picture. For the particular parameter values considered in Figure 9, the indifference point occurs at $\hat{c} \approx 0.61$. Although it is also profitable to invest in $\mathrm{R} \& \mathrm{D}$ for some initial technology level $c_{0} \in(\hat{c}, 1)$, the technology is not promising enough for the monopolist to select the stable path towards the saddlepoint steady-state. Rather, he invests in R\&D at some smaller rate that retards the decay of the technology level optimally; but eventually the technology will leave the market, and the monopolist with it. ${ }^{29}$

For an even higher initial level of marginal cost $\left(c_{0} \geq 1\right)$, the monopolist does not initiate any activity; the new technology is not developed at all and the monopolist does not enter the market. ${ }^{30}$

Utterback (1994) documents several examples of technologies where process innovations continue to be realized although it is known that these technologies will leave the market in the near future. For instance, steam-powered saws and conveyors were first used in the ice harvesting industry after the introduction of machine-made ice. And self-setting shutters, small plate cameras, and celluloid substitutes for glass were introduced in the dry plate photography at the time that Kodak introduced roll film cameras. These examples correspond well to the scenario predicted by the "exit trajectory".

Region III is similar to Region II in that for $c_{0} \geq 1$ the firm does not initiate any activity and only for sufficiently low initial marginal costs $c_{0} \in\left(0, c_{R}\right)$ there is conver-

\footnotetext{
${ }^{29}$ Note that if $c_{0} \in(\hat{c}, 1)$, the alternative strategy of producing but not investing anything in $\mathrm{R} \& \mathrm{D}$, which corresponds to "following" the $c$-axis below the exit trajectory in Figure 9, yields lower total discounted profits than following the exit trajectory (recall Lemma 4).

${ }^{30}$ Conjugate points $P_{1}$ and $P_{2}$ indicate the last element on the respective trajectory (when starting from its end) where the marginal cost is still increasing/decreasing. Considering a particular trajectory beyond this point would give us two possible $k$-points for each $c$, of which the one beyond the $P$ point is suboptimal. Observe that in the plot of the profits, the value of the total profit for the exit trajectory (starting from its end) increases all along the way to $P_{2}$ (where it obtains a cusp point), beyond which it starts decreasing.
} 

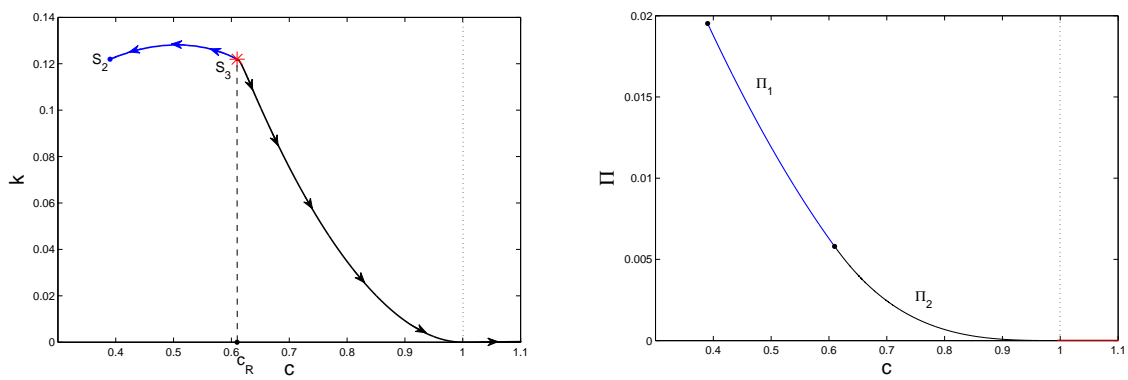

Figure 10: State-control space and the total profit functions when there is a repeller point $\left(c_{R}\right)$ in the region with positive production. The plots are drawn for parameter values $(\rho, \phi)=(4,8.2)$. The stable path of the saddle-point steady state and the exit trajectory both flow out of a repelling point, which is the unstable node $S_{3}$ (corresponding to $c=0.6098$ ).

gence to the saddle-point steady state. There is, however, a small qualitative difference between the two regions: in Region III, the threshold point is not an indifference point but a repeller (see Figure 10). Starting close to the repeller, the firm will "linger" near to it for a long time before deciding on developing the technology (if $c_{0}<c_{R}$ ), or on phasing it out eventually (if $c_{0}>c_{R}$ ). ${ }^{31}$

Region III is separated from Region II by a type-2 indifference-repeller bifurcation curve $\left(\operatorname{IR}_{1}(2)\right)$, at which a repeller changes into an indifference point. ${ }^{32}$ Fixing $\phi$ and varying $\rho$, it can be shown that the exit trajectory of a repeller lies below that of an indifference point. ${ }^{33}$ This is also intuitive: a lower discount rate $\rho$ (in the case of an indifference point) implies larger investments in $\mathrm{R} \& \mathrm{D}$ as the monopolist values future profits relatively more. Hence, for each initial value of marginal cost, the exit trajectory corresponding to a lower discount rate lies above the exit trajectory corresponding to a higher discount rate.

\subsection{Regions IV and V}

Region IV is separated from Region III by a saddle-node bifurcation curve, given by $\phi=4 \sqrt{\rho}$. This curve represents parameter combinations for which the saddle and the unstable node $S_{3}$ coincide and form a semi-stable steady state. After the bifurcation, both the saddle and the unstable node disappear. In such a case (see Figure 11), the

\footnotetext{
${ }^{31}$ Observe in Figure 10 that in the case of a repeller, the two trajectories never occur at the same time. Hence, the corresponding total profit functions (the right-hand picture) do not intersect.

${ }^{32}$ Before the bifurcation (Region $I I$ ), the unstable steady state $\left(S_{3}\right)$ has two complex eigenvalues with positive real parts. The stable path and the exit trajectory both spiral out from it, which yields an indifference point to the left of the unstable steady state (focus). At the bifurcation, both eigenvalues become real and equal, such that all trajectories move away from the unstable steady state in the direction of a unique eigenvector. After the bifurcation, the steady state has two real positive eigenvalues and two corresponding (and distinct) eigenvectors.

${ }^{33}$ Fixing $\rho$ and varying $\phi$ leads to the same conclusion.
} 

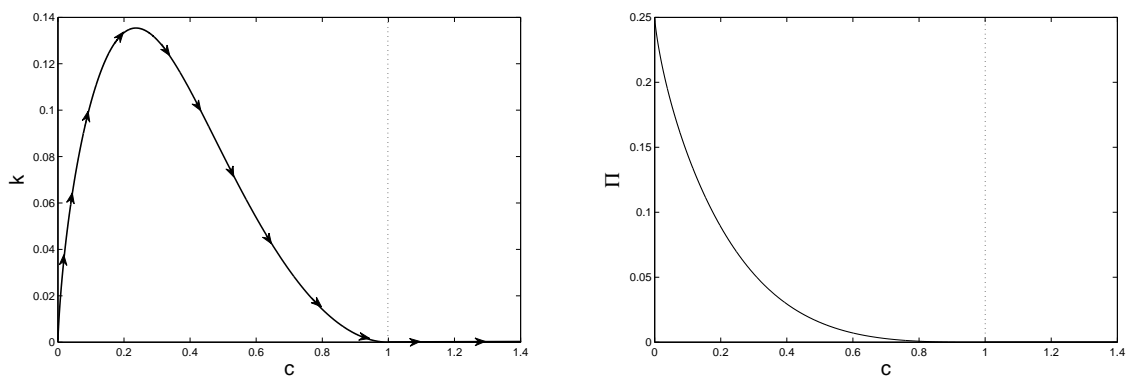

Figure 11: State-control space and the total profit function for the case with no saddlepoint steady state. Parameter values are $(\rho, \phi)=(1,3)$. In this situation (and any other situation in which $\phi<4 \sqrt{\rho}$ ), the exit trajectory covers the whole space and it is the only solution. Observe that the total discounted profit is positive for $c<1$.

only solution left is the exit trajectory: for $c_{0} \geq 1$ the firm never initiates any activity, while for $c_{0} \in(0,1)$ the firm does invest in R\&D but it will leave the market at some future instant. This is again intuitive as in Region $I V$ we have that $\phi$ is relatively small (for a given $\rho$ ). That is, $R \& D$ is relatively inefficient and/or market demand is relatively small. Note that in Figure $11, \mathrm{R} \& \mathrm{D}$ investments are initially increasing over time for very low values of marginal costs. These investments reduce the pace at which the monopolist will leave the market; for low initial marginal cost it is profitable to slow down this process in order to profit optimally from the relatively favorable initial technology.

Finally, Region $V$ resembles Region $I V$ in that only the exit trajectory is optimal (see Figure 12). This is in spite of the fact that points in Region $V$ have higher $\phi$ values than points in Region $I V$ with the same value of $\rho$, and that the saddle-point steady state $\left(S_{2}\right)$ together with its stable path (denoted by $T_{1}$ ) exists. A distinctive feature of Region $V$ is that the exit trajectory (denoted by $T_{2}$ ) intersects the vertical line through the saddlepoint steady state. Whenever this is the case, it is never optimal to be at the saddle, or to follow any stable path leading towards it. Rather, the exit trajectory is optimal, yielding a higher discounted profit flow: that is, the value of the Hamiltonian at any point of the exit trajectory is higher than at points with the same value of marginal cost on the stable path. ${ }^{34}$

Observe that in the upper right graph of Figure 12 total discounted profit evaluated along the stable path $\left(\Pi_{1}\right)$ is always below that evaluated along the exit trajectory $\left(\Pi_{2}\right)$. For the sake of completeness, the second stable path (denoted by $T_{4}$ ) and the unstable path (denoted by $T_{3}$ ) are plotted jointly with the corresponding total discounted profit functions $\left(\Pi_{4}\right.$ and $\Pi_{3}$, respectively). The latter are also both lower than $\Pi_{2}$. Therefore,

\footnotetext{
${ }^{34}$ This follows from the fact that the derivative of the Hamilton function with respect to $k$ is negative for $k<1 / \phi$ (which is also the $k$-coordinate of the saddle-point steady state).
} 

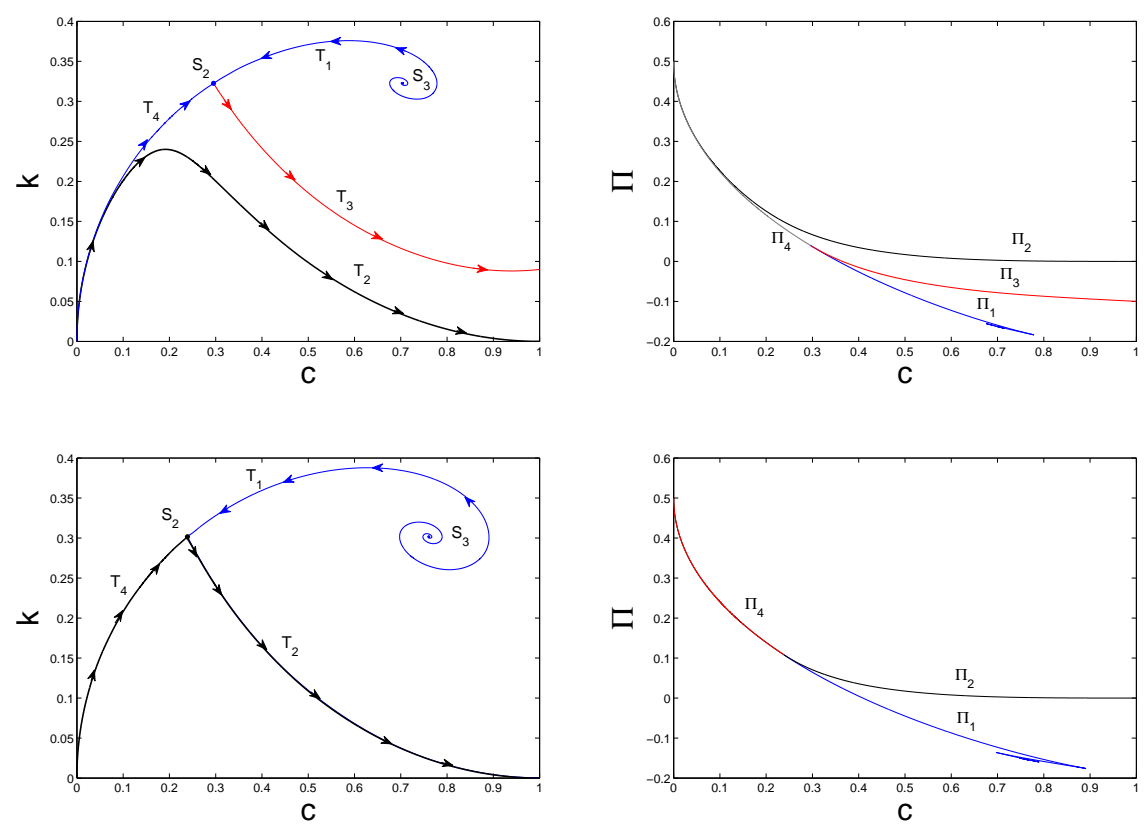

Figure 12: State-control space and the total profit functions before and at the indifference-attractor bifurcation. The plots in the upper two graphs (before the bifurcation) are drawn for $(\rho, \phi)=(0.5,3.1)$. The plots in the lower two graphs show that the bifurcation occurs at $(\rho, \phi) \approx(0.5,3.32)$.

as said, for parameter values in Region $V$, the exit trajectory is always optimal and the saddle-point steady state is never approached, neither from above nor from below. ${ }^{35}$ Put differently, the saddle-point steady state is not even locally optimal.

The $\mathrm{IA}_{1}$ curve that bounds Region $V$ from above is an indifference-attractor bifurcation curve. At the $\mathrm{IA}_{1}$ bifurcation (depicted in the second row of Figure 12), the exit trajectory $\left(T_{2}\right)$ coincides with the unstable path $\left(T_{3}\right)$ and yields a higher total discounted profit than the stable path converging to the saddle-point steady state from the right (see also Kiseleva and Wagener (2011) and Wagener (2003)). Hence, there is no convergence to the saddle from above. However, the exit trajectory does not intersect any vertical line in the region to the left of the saddle-point steady state. Consequently, the left-hand stable path remains the only candidate for an optimal solution. That is, for initial marginal cost lower than the saddle-point steady-state value, the optimal trajectory approaches the saddle-point steady state from below. ${ }^{36}$

\footnotetext{
${ }^{35}$ The second stable path is not drawn in the previous figures so as not to blur the exposition. The policy that it induces implies that the long-run optimal marginal cost is just higher than the initial value of the marginal cost.

${ }^{36}$ Notice that for the values of initial marginal cost lower than the saddle-point steady state, the saddle is also approached from below for parameter values in Regions I, II, and III as the left-hand stable path of the saddle point remains the only candidate for the optimal solution in the respective region.
} 
All in all, before the bifurcation, the exit trajectory lies below the unstable path of the saddle; after the bifurcation, it lies above it. At the bifurcation, the two trajectories change their relative positions, the saddle-point steady state becomes locally optimal and an indifference point is created. That is, we enter Region II. In other words, moving from Region $V$, where the firm always exits the market, by increasing $\phi$ while keeping $\rho$ fixed, we arrive at Region II, where staying in the market might be an optimal solution, depending on the initial level $c_{0}$ of the marginal cost. The interpretation is straightforward: staying in the market becomes an option if $\mathrm{R} \& \mathrm{D}$ becomes more efficient and/or if market demand increases.

The curve bounding Region $V$ from below is an inessential saddle-node bifurcation curve $\left(\mathrm{SN}_{1}^{\prime}\right)$. It is similar to the $\mathrm{SN}_{1}$ curve in that two new steady states appear when crossing it. However, it is inessential for the optimization problem: the bifurcation does not entail a qualitative change of the optimal strategy, as the steady states involved in the $\mathrm{SN}_{1}^{\prime}$ bifurcation are not associated with the optimal strategy; as above, the exit trajectory below the unstable path of the saddle remains the optimal trajectory. Consequently, while the state-control system does bifurcate, the structure of the optimal solutions does not.

Together, Region $I V$ and Region $V$ represent the parameter region for which there is never convergence to the saddle-point steady state because it is either not optimal to approach it (Region $V$ ) or because there is no saddle-point steady state at all (Region IV). ${ }^{37}$

\section{Concluding remarks}

Existing models of $\mathrm{R} \& \mathrm{D}$ are difficult to reconcile with empirical aspects of R\&D. These include the observation that many initial technologies ("prototypes" or "ideas") need to be developed further before they can enter the market, that only a minority of initial technologies is successfully brought to the market, that production starts only after an initial stage during which the technology is developed further, and that process innovations are implemented for technologies that are destined to leave the market. In this paper, we have developed a dynamic model of R\&D that describes the R\&D investment decision of a monopolist, and that is better aligned with these empirical observations.

A distinguishing feature of our analysis is that we provide a global analysis, as opposed to all related papers. Accordingly, we do not limit initial technologies to carry marginal costs that are below the choke price. The part of the parameter space for

\footnotetext{
${ }^{37}$ Cellini and Lambertini (2009) restrict the values of the marginal cost to $c \in[0,1]$ and assume that the saddle-point steady state is always optimally approached. Clearly, their conclusions hold only for parameters in Region $I$ as only there we have convergence to the steady state for initial marginal costs $c \in(0,1]$. A look at Figure 5, however, reveals that four other regions exist where the convergence is not guaranteed for all $c \in(0,1]$.
} 
which such technologies could eventually lead to the saddle-point steady state is not negligible indeed. More generally, we have shown that there always exists a critical value of initial marginal cost above which the firm does not initiate any (R\&D) activity; the saddle-point steady state is never globally optimal.

Our analysis suggests a careful look at competition policies that reduce mark-ups in products markets. Reduced future profits diminish the profitability to develop further initial technologies, in particular those that come with marginal costs above the choke price. Not developing further these technologies does not surface as a cost of competition policy as there is no production yet that will be taken from the market. Obviously, a proper assessment of a competition policy requires an analysis that includes firm competition, an exercise that is part of our future research agenda.

\section{References}

[1] ABERNATHY, W. J. and UTTERBACK, J. M. (1975), "A Dynamic Model of Product and Process Innovation", Omega, 3, 639-656.

[2] ABERNATHY, W. J. and UTTERBACK, J. M. (1978), "Patterns of Industrial Innovation", Technology Review, 80, 40-47.

[3] ADAMS, J. and GRILICHES, Z. (1996), "Measuring Science: An Exploration", Proceedings of the National Academy of Sciences, 93, 12664-12670.

[4] CELliNI, R. and LAMBERTINI, L. (2009), "Dynamic R\&D with Spillovers: Competition vs Cooperation", Journal of Economic Dynamics \& Control, 33, $568-582$.

[5] D' ASPREMONT, C. and JACQUEMIN, A. (1988), "Cooperative and Noncooperative R\&D in Duopoly with Spillovers”, The American Economic Review, 78, $1133-1137$.

[6] DE BONDT, R. (1997), "Spillovers and Innovative Activities", International Journal of Industrial Organization, 15, 1-28.

[7] DORASZELSKI, U. (2004), "Innovations, Improvements, and the Optimal Adoption of New Technologies", Journal of Economic Dynamics \& Control, 28, 1461-1480.

[8] GRASS, D., CAUlkins, J. P., FEICHTINGER, G., TRAGleR G. and BEHRENS, D. A. (2008), Optimal Control of Nonlinear Processes (SpringerVerlag). 
[9] GRAVES, S. B. and LANGOWITZ, N. S. (1993), "Innovative Productivity and Returns to Scale in the Pharmaceutical Industry", Strategic Management Journal, 14, 593-605.

[10] HOPPE, H. C. (2002), "The Timing of New Technology Adoption: Theoretical Models and Empirical Evidence”, The Manchester School, 70, 56-76.

[11] JUDD, K. L. (1998), Numerical Methods in Economics (Cambridge, MA: The MIT Press).

[12] KAMIEN, M. I., MULLER, E. and ZANG, I. (1992), "Research Joint Ventures and R\&D Cartels", The American Economic Review, 82, 1293-1306.

[13] KAMIHIGASHI, T. (2001), "Necessity of Transversality Condition for Infinite Horizon Problems”, Econometrica, 69, 995-1012.

[14] KELLEY, W. G. and PETERSON, A. C. (2010), The Theory of Differential Equations: Classical and Qualitative, 2nd Edition (Springer).

[15] KISELEVA, T. and WAGENER, F. O. O. (2010), "Bifurcations of Optimal Vector Fields in the Shallow Lake Model”, Journal of Economic Dynamics \& Control, 34, 825-843.

[16] KISELEVA, T. and WAGENER, F. O. O. (2011), "Bifurcations of Optimal Vector Fields", CeNDEF Working paper, University of Amsterdam.

[17] KOVAC, E., VINOGRADOV, V. and ZIGIC, K. (2010), “Technological Leadership and Persistence of Monopoly Under Endogenous Entry: Static Versus Dynamic Analysis", Journal of Economic Dynamics and Control, 34, 1421-1441.

[18] LAMBERTINI, L. and MANTOVANI, A. (2009), "Process and Product Innovation by a Multiproduct Monopolist: A Dynamic Approach", International Journal of Industrial Organization, 27, 508-518.

[19] LEE, T. and WILDE, L. L. (1980), "Market Structure and Innovation: A Reformulation”, Quarterly Journal of Economics, 94, 429-436.

[20] LOURY, G. C. (1979), "Market Structure and Innovation”, Quarterly Journal of Economics, 93, 395-410.

[21] MADSEN, J. B. (2007), “Are There Diminishing Returns to R\&D?” Economic Letters, 95, 161-166.

[22] MICHEL, P. (1982), "On the Transversality Condition in Infinite Horizon Optimal Problems”, Econometrica, 50, 975-985. 
[23] POLASKY, S., DE ZEEUW, A. and WAGENER, F. O. O. (2011), “Optimal Management with Potential Regime Shifts", Journal of Environmental Economics and Management, 62, 229-240.

[24] QIU, L. D. (1997), “On the Dynamic Efficiency of Bertrand and Cournot Equilibria", Journal of Economic Theory, 75, 213-229.

[25] REINGANUM, J. F. (1989), “The Timing of Innovation: Research, Development, and Diffusion", Handbook of Industrial Organization, 1, 849-908.

[26] SCHWARTZMAN, D. (1976), Innovation in the Pharmaceutical Industry (Baltimore: John Hopkins University Press).

[27] SOHRAB, H. H. (2003), Basic Real Analysis (Boston: Birkhäuser).

[28] STALFORD, H. (1971), "Sufficient Conditions for Optimal Control with State and Control Constraints", Journal of Optimization Theory and Applications, 7, $118-135$.

[29] STOBAUGH, R. (1988), Innovation and Competition: The Global Management of Petrochemical Products (Boston: Harvard Business School Press).

[30] UTTERBACK, J. M. (1994), Mastering the Dynamics of Innovation. How Companies Can Seize Opportunities in the Face of Technological Change. (Boston: Harvard Business School Press).

[31] VERNON, J. M. and GUSEN, P. (1974), “Technical Change and Firm Size: The Pharmaceutical Industry”, Review of Economics and Statistics, 56, 294-302.

[32] WAGENER, F. O. O. (2003), "Skiba Points and Heteroclinic Bifurcations, with Applications to the Shallow Lake System”, Journal of Economic Dynamics \& Control, 27, 1533-1561. 


\section{Appendices}

\section{A Proof of Lemma 1}

A rescaled variable or parameter is distinguished by a tilde: for instance, if $\pi$ denotes profit, then $\tilde{\pi}$ denotes profit in rescaled variables. The profit function in the original (non-rescaled) model is:

$$
\pi=(A-q-c) q-b k^{2}
$$

Using the conversion rules given in Lemma 1, we obtain:

$$
\begin{aligned}
\pi & =(A-q-c) q-b k^{2} \\
& =(A-A \tilde{q}-A \tilde{c}) A \tilde{q}-b\left(\frac{A}{\sqrt{b}} \tilde{k}\right)^{2} \\
& =A^{2}\left((1-\tilde{q}-\tilde{c}) \tilde{q}-\tilde{k}^{2}\right) \\
& =A^{2} \tilde{\pi}
\end{aligned}
$$

The equation for the evolution of marginal cost over time is in original variables given by:

$$
\dot{c}(t)=c(t)(-k(t)+\delta) .
$$

Write $c(t)=c\left(\frac{1}{\delta} \tilde{t}\right)$. Then

$$
\begin{aligned}
\frac{d c}{d \tilde{t}} & =\frac{d c}{d t} \frac{d t}{d \tilde{t}} \\
& =\frac{\dot{c}}{\delta} \\
& =\left(1-\frac{1}{\delta} k\right) c .
\end{aligned}
$$

Setting $k=\frac{A}{\sqrt{b}} \tilde{k}$ and substituting it in the previous equation, we obtain:

$$
\frac{d c}{d \tilde{t}}=\left(1-\frac{A}{\delta \sqrt{b}} \tilde{k}\right) c
$$

It is now natural to introduce $\phi=\frac{A}{\delta \sqrt{b}}$.

Note that if $\tilde{c}=c / A$, then $\dot{\tilde{c}}=\dot{c} / A$ and

$$
\dot{\tilde{c}}=(1-\phi \tilde{k}) \tilde{c}
$$

Observe finally that if $t=\frac{\tilde{t}}{\delta}$, then $e^{-\tilde{\rho} \tilde{t}}=e^{-\rho t}$ if and only if $\tilde{\rho}=\frac{\rho}{\delta}$. 


\section{B Proof of Lemma 2}

As $\rho>0$, the equilibrium condition $\dot{k}=\rho k=0$ implies that $k=0$. But then $\dot{c}=c(1-\phi k)=c$. This is equal to 0 if, and only if, $c=0$. But this cannot be, as in the system with zero production we have $c \geq 1$.

\section{Proof of Proposition 1}

Assume $\phi>4 \sqrt{\rho}$. The stability of the steady states can be analyzed by evaluating the trace and determinant of the following Jacobian matrix: ${ }^{38}$

$$
J^{M}=\left[\begin{array}{cc}
\frac{\partial \dot{c}}{\partial c} & \frac{\partial \dot{c}}{\partial k} \\
\frac{\partial \dot{k}}{\partial c} & \frac{\partial \dot{k}}{\partial k}
\end{array}\right]=\left[\begin{array}{cc}
1-\phi k & -\phi c \\
-\frac{\phi(1-2 c)}{4} & \rho
\end{array}\right]
$$

At $c=k=0$, the trace $\tau$ of the matrix $J^{M}$ is given as

$$
\tau \stackrel{\text { def }}{=} \operatorname{tr} J^{M}=1+\rho>0
$$

its determinant $\Delta$ is

$$
\Delta \stackrel{\text { def }}{=} \operatorname{det} J^{M}=\rho>0
$$

and its discriminant $D$ is

$$
D \stackrel{\text { def }}{=} \tau^{2}-4 \Delta=(1-\rho)^{2}>0
$$

Hence, this steady state is an unstable node.

Evaluating the Jacobian matrix at

$$
k=\frac{\phi}{4 \rho} c(1-c), \quad c=\frac{1}{2}+V=\frac{1}{2}+\frac{1}{2} \sqrt{1-\frac{16 \rho}{\phi^{2}}},
$$

we obtain $\tau=\rho>0$ and

$$
\Delta=\frac{\sqrt{\phi^{2}-16 \rho}\left[\phi+\sqrt{\phi^{2}-16 \rho}\right]}{8}=\phi^{2} V\left(\frac{1}{4}+\frac{1}{2} V\right),
$$

which is clearly positive if $\phi>4 \sqrt{\rho}$. Hence, this steady state is also unstable. The

\footnotetext{
${ }^{38}$ Note that the trace of the respective Jacobian matrix is equal to the sum of eigenvalues, while its determinant is equal to their product. If the real part of each eigenvalue is negative, then the steady state is asymptotically stable. If the real part of at least one of the eigenvalues is positive, then the steady state is unstable. More particular, if one eigenvalue is real and positive and the other one real and negative, the steady state is said to be a saddle. In this last situation, there are four special trajectories, the separatrices, two of which are forward asymptotic to the saddle, while the other two are backward asymptotic to it. The union of the former separatrices with the saddle form the stable manifold of the saddle; analogously, the union of the latter with the saddle form the unstable manifold.
} 
discriminant takes the value

$$
D=\rho(8+\rho)-\frac{1}{2} \phi\left(\phi+\sqrt{\phi^{2}-16 \rho}\right),
$$

which is zero for $\phi=\phi_{0}=\sqrt{\frac{\rho(8+\rho)^{2}}{(4+\rho)}}$, negative for $\phi>\phi_{0}$, and positive for $4 \sqrt{\rho} \leq$ $\phi<\phi_{0}$. The steady state is an unstable node if $D>0$ and an unstable focus if $D<0$. In the latter case, the eigenvalues of $J^{M}$ are complex conjugates with positive real parts. ${ }^{39}$

Finally, evaluating the Jacobian matrix at

$$
k=\frac{\phi}{4 \rho} c(1-c), \quad c=\frac{1}{2}-V=\frac{1}{2}-\frac{1}{2} \sqrt{1-\frac{16 \rho}{\phi^{2}}},
$$

we obtain $\tau=\rho>0$ and

$$
\Delta=\frac{\phi^{2}-16 \rho-\phi \sqrt{\phi^{2}-16 \rho}}{8} .
$$

If $\phi>4 \sqrt{\rho}$, then $\Delta<0$, and the eigenvalues are real and have opposite sign. Therefore, (48) is a saddle-point steady state of the system. Observe that for $\phi=4 \sqrt{\rho}$, the two steady states (45) and (48) coincide at $c=1 / 2$ and $k=\frac{1}{\phi}$. A saddle-node bifurcation occurs at these parameter values, where the two equilibria collide and disappear. ${ }^{40}$

Substituting the expression for the steady-state marginal cost of the steady states other than the origin into (32), the expression for the optimal investment in the steady state simplifies to $k^{M}=\frac{1}{\phi}$.

\section{Proof of Lemma 3}

As we deal with both versions of the model, we use tildes in the notation of rescaled variables and parameters to avoid ambiguity. We first prove the stated relations between parameters and steady-state values for the rescaled model. Take $\phi>4 \sqrt{\tilde{\rho}}$, such that the saddle-point steady state exists. The saddle-point steady state values of the marginal cost, output, and profit are given in (36)-(38). Consumer surplus and total surplus are, respectively:

$$
\begin{gathered}
\widetilde{C S}^{M}=\frac{(1-\tilde{p}) \tilde{q}}{2}=\frac{1}{2}\left(\tilde{q}^{M}\right)^{2}=\frac{1}{32}(1+2 V)^{2}, \\
\widetilde{T S}^{M}=\tilde{\pi}^{M}+\widetilde{C S}^{M}=\frac{3}{32}(1+2 V)^{2}-\frac{1}{\phi^{2}} .
\end{gathered}
$$

\footnotetext{
${ }^{39}$ Note that the eigenvalues of $J^{M}$ are $r_{1,2}=\frac{1}{2}\left(\tau \pm \sqrt{\tau^{2}-4 \Delta}\right)$.

${ }^{40}$ In Figure 3, this corresponds to the locus $\dot{c}=0$, which is the horizontal line $k=\frac{1}{\phi}$, becoming tangent to the locus $\dot{k}=0$ at its peak.
} 
Substituting the expression for $V$ given in (35) in the steady state expressions (36)-(38) and (50)-(51) and taking appropriate derivatives, we obtain:

$$
\begin{aligned}
& \frac{\partial \tilde{c}^{M}}{\partial \phi}=-\frac{8 \tilde{\rho}}{\phi^{2} \sqrt{\phi^{2}-16 \tilde{\rho}}}<0, \\
& \frac{\partial \tilde{c}^{M}}{\partial \tilde{\rho}}=\frac{4}{\phi \sqrt{\phi^{2}-16 \tilde{\rho}}}>0, \\
& \frac{\partial \tilde{q}^{M}}{\partial \phi}=\frac{4 \tilde{\rho}}{\phi^{2} \sqrt{\phi^{2}-16 \tilde{\rho}}}>0, \\
& \frac{\partial \tilde{q}^{M}}{\partial \tilde{\rho}}=-\frac{2}{\phi \sqrt{\phi^{2}-16 \tilde{\rho}}}<0, \\
& \frac{\partial \tilde{\pi}^{M}}{\partial \phi}=\frac{2\left(1+\tilde{\rho}+\frac{\tilde{\rho} \phi}{\sqrt{\phi^{2}-16 \tilde{\rho}}}\right)}{\phi^{3}}>0, \\
& \frac{\partial \tilde{\pi}^{M}}{\partial \tilde{\rho}}=-\frac{1+\frac{\phi}{\sqrt{\phi^{2}-16 \tilde{\rho}}}}{\phi^{2}}<0,
\end{aligned}
$$

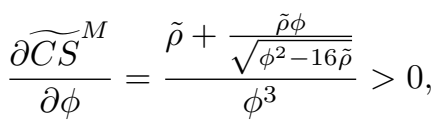

$$
\begin{aligned}
& \frac{\partial \widetilde{C S}^{M}}{\partial \tilde{\rho}}=-\frac{1+\frac{\phi}{\sqrt{\phi^{2}-16 \tilde{\rho}}}}{2 \phi^{2}}<0, \\
& \frac{\partial \widetilde{T S}}{\partial \phi}=\frac{2+3 \tilde{\rho}+\frac{3 \tilde{\rho} \phi}{\sqrt{\phi^{2}-16 \tilde{\rho}}}}{\phi^{3}}>0, \\
& \frac{\partial \widetilde{T S}^{M}}{\partial \tilde{\rho}}=-\frac{3+\frac{3 \phi}{\sqrt{\phi^{2}-16 \tilde{\rho}}}}{2 \phi^{2}}<0 .
\end{aligned}
$$

All inequalities follow straightforwardly form the admissible values of the parameters.

We now prove the stated relations for the original model. Applying the conversion rules defined in Lemma 1, we obtain:

$$
\begin{aligned}
c^{M} & =\frac{A-\sqrt{A^{2}-16 b \delta \rho}}{2}, \\
q^{M} & =\frac{A+\sqrt{A^{2}-16 b \delta \rho}}{4}, \\
\pi^{M} & =\frac{A^{2}-8 b \delta(\delta+\rho)+A \sqrt{A^{2}-16 b \delta \rho}}{8}, \\
C S^{M} & =\frac{1}{2}\left(q^{M}\right)^{2}=\frac{1}{32}\left(A+\sqrt{A^{2}-16 b \delta \rho}\right)^{2}, \\
T S^{M} & =\frac{3 A^{2}-8 b \delta(2 \delta+3 \rho)+3 A \sqrt{A^{2}-16 b \delta \rho}}{16} .
\end{aligned}
$$


Take $A>4 \sqrt{b \delta \rho}$, such that the saddle-point steady state exists. Then:

$$
\begin{gathered}
\frac{\partial c^{M}}{\partial b}=\frac{4 \delta \rho}{\sqrt{A^{2}-16 b \delta \rho}}>0, \\
\frac{\partial c^{M}}{\partial \delta}=\frac{4 b \rho}{\sqrt{A^{2}-16 b \delta \rho}}>0, \\
\frac{\partial q^{M}}{\partial b}=-\frac{2 \delta \rho}{\sqrt{A^{2}-16 b \delta \rho}}<0, \\
\frac{\partial q^{M}}{\partial \delta}=-\frac{2 b \rho}{\sqrt{A^{2}-16 b \delta \rho}}<0 .
\end{gathered}
$$

It follows from the definition of consumer surplus $\left(C S^{M}=\frac{1}{2}\left(q^{M}\right)^{2}\right)$ that the effect of $b$ and $\delta$ on $C S^{M}$ is qualitatively the same as their effect on $q^{M}$. Moreover, we have:

$$
\begin{gathered}
\frac{\partial \pi^{M}}{\partial b}=-\delta(\delta+\rho)-\frac{A \delta \rho}{\sqrt{A^{2}-16 b \delta \rho}}<0, \\
\frac{\partial \pi^{M}}{\partial \delta}=b\left(-2 \delta+\rho\left(-1-\frac{A}{\sqrt{A^{2}-16 b \delta \rho}}\right)\right)<0, \\
\frac{\partial T S^{M}}{\partial b}=\frac{1}{2} \delta\left(-2 \delta+\rho\left(-3-\frac{3 A}{\sqrt{A^{2}-16 b \delta \rho}}\right)\right)<0, \\
\frac{\partial T S^{M}}{\partial \delta}=\frac{1}{2} b\left(-4 \delta+\rho\left(-3-\frac{3 A}{\sqrt{A^{2}-16 b \delta \rho}}\right)\right)<0 .
\end{gathered}
$$

Again, all inequalities follow straightforwardly from the relevant values of the parameters.

\section{E Proof of Remark 1}

The Hessian matrix of the Pontryagin function with respect to control and state variables is

$$
D_{(q, k, c)}^{2} P=\left[\begin{array}{ccc}
\frac{\partial^{2} P(\cdot)}{\partial q^{2}} & \frac{\partial^{2} P(\cdot)}{\partial q \partial k} & \frac{\partial^{2} P(\cdot)}{\partial q \partial c} \\
\frac{\partial^{2} P(\cdot)}{\partial k \partial q} & \frac{\partial^{2} P(\cdot)}{\partial k^{2}} & \frac{\partial^{2} P(\cdot)}{\partial k \partial c} \\
\frac{\partial^{2} P(\cdot)}{\partial c \partial q} & \frac{\partial^{2} P(\cdot)}{\partial c \partial k} & \frac{\partial^{2} P(\cdot)}{\partial c^{2}}
\end{array}\right]=\left[\begin{array}{ccc}
-2 & 0 & -1 \\
0 & -2 & -\phi \lambda \\
-1 & -\phi \lambda & 0
\end{array}\right] .
$$

The leading principal minor of the first order of this matrix is -2 . The leading principal minor of the third order, which is also the determinant, of the above matrix is equal to $2+2 \phi^{2} \lambda^{2}$. As the leading principal minors of odd order do not have the same sign, the matrix is indefinite. Consequently, the Pontryagin function is nowhere jointly concave 
in state and control variables. Hence, the Arrow-Mangasarian sufficiency conditions are not satisfied. ${ }^{41}$

\section{F Proof of Lemma 4}

To prove the lemma, we consider the state-co-state form of the solution, given by:

$$
\begin{aligned}
& \dot{c}=\left\{\begin{aligned}
c\left(1+\frac{1}{2} \phi^{2} \lambda c\right), & 0 \leq c<1, \lambda \leq 0 \\
c, & 0 \leq c<1, \lambda>0 ; \\
c\left(1+\frac{1}{2} \phi^{2} \lambda c\right), & c \geq 1, \lambda \leq 0 \\
c, & c \geq 1, \lambda>0 ;
\end{aligned}\right. \\
& \dot{\lambda}=\left\{\begin{array}{rrr}
\frac{1}{2}(1-c)+\left(\rho-1-\frac{1}{2} \phi^{2} \lambda c\right) \lambda, & 0 \leq c<1, \lambda \leq 0 ; \\
\frac{1}{2}(1-c)+(\rho-1) \lambda, & 0 \leq c<1, \lambda>0 ; \\
\left(\rho-1-\frac{1}{2} \phi^{2} \lambda c\right) \lambda, & c \geq 1, \lambda \leq 0 ; \\
(\rho-1) \lambda, & c \geq 1, \lambda>0 ;
\end{array}\right. \\
& H(c, \lambda)=\left\{\begin{array}{rr}
\frac{1}{4}(1-c)^{2}+\frac{1}{4} \phi^{2} \lambda^{2} c^{2}+\lambda c, & 0 \leq c<1, \lambda \leq 0 ; \\
\frac{1}{4}(1-c)^{2}+\lambda c, & 0 \leq c<1, \lambda>0 ; \\
\frac{1}{4} \phi^{2} \lambda^{2} c^{2}+\lambda c, & c \geq 1, \lambda \leq 0 ; \\
\lambda c, & c \geq 1, \lambda>0 .
\end{array}\right.
\end{aligned}
$$

Introduce the characteristic function $\chi_{S}$ of a set $S$ by

$$
\chi_{S}(x)=\left\{\begin{array}{lll}
1 & \text { if } & x \in S \\
0 & \text { if } & x \notin S,
\end{array}\right.
$$

differential equations (57) and (58) can be rewritten more compactly as:

$$
\dot{c}=c+\chi_{(-\infty, 0]}(\lambda) \frac{1}{2} \phi^{2} \lambda c^{2} \stackrel{\text { def }}{=} F(c, \lambda)
$$

and

$$
\dot{\lambda}=(\rho-1) \lambda-\chi_{(-\infty, 0]}(\lambda) \frac{1}{2} \phi^{2} c \lambda^{2}+\chi_{[0,1)}(c) \frac{1}{2}(1-c) \stackrel{\text { def }}{=} G(c, \lambda) .
$$

The state-co-state space is presented in Figure 13. We first consider the trajectories in the region where $c \geq 1$ and $\lambda>0$, which are the solution to the following canonical

\footnotetext{
${ }^{41}$ For details of sufficiency conditions, see, for instance, Grass et al. (2008).
} 


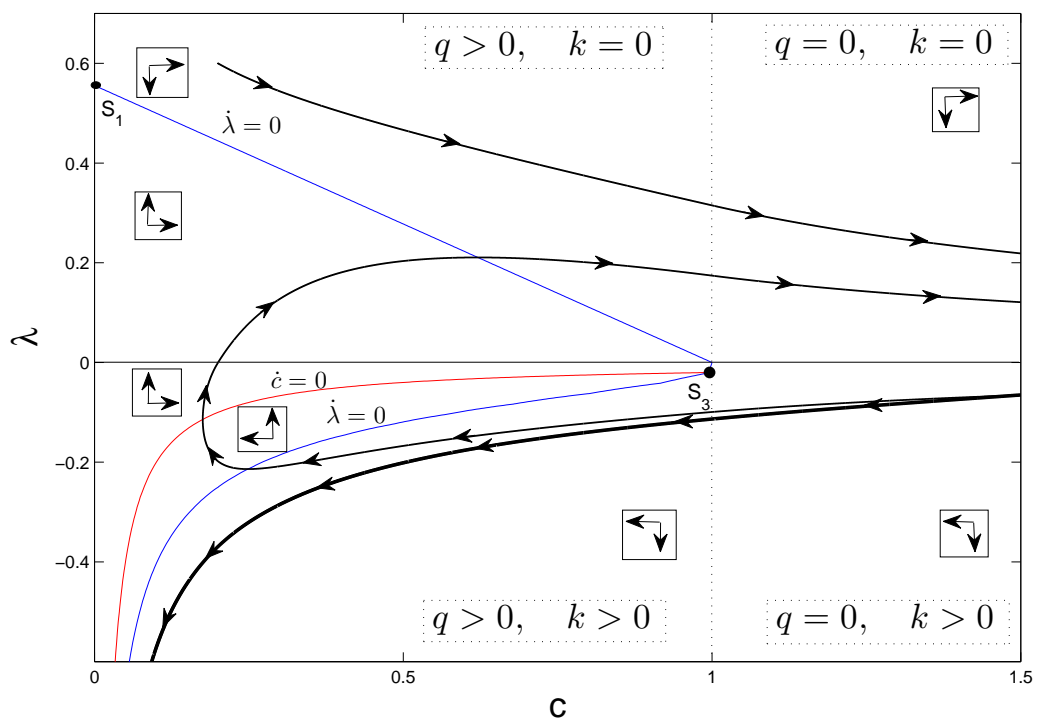

Figure 13: Illustrative sketch of the state-co-state space (for $\rho<1$ ). The dotted vertical line $c=1$ separates the region with zero production from the region with a positive level of production, whereas the horizontal line $\lambda=0$ separates the region with positive investment from the region with zero investment. The loci $\dot{c}=0$ and $\dot{\lambda}=0$ intersect in the two unstable steady states $\left(S_{1}\right.$ and $\left.S_{3}\right)$ and the saddle-point steady state $S_{2}$ (not indicated). A number of trajectories is indicated by black curves: the arrows point in the direction of the flow. The thick curve indicates the stable path leading to the saddle-point steady state.

system:

$$
\left\{\begin{array}{l}
\dot{c}=c, \\
\dot{\lambda}=(\rho-1) \lambda,
\end{array}\right.
$$

given by $\lambda(t)=C_{1} e^{(\rho-1) t}$ and $c(t)=C_{2} e^{t}$, where $C_{1}$ and $C_{2}$ are positive constants. As mentioned in the main text, every candidate for an optimal solution must necessarily satisfy the transversality condition $\lim _{t \rightarrow \infty} e^{-\rho t} \lambda(t) c(t)=0$. As we now show, the trajectories in the region considered violate this condition. Moreover, they also violate the following transversality condition: ${ }^{42}$

$$
\lim _{t \rightarrow \infty} e^{-\rho t} H(c, \lambda)=0,
$$

which in this case coincides with the first transversality condition, as shown below. It follows from Michel (1982) that condition (64) is also a necessary condition, and hence that it allows exclusion of trajectories which verify the other necessary conditions in an

\footnotetext{
${ }^{42}$ In words, the above condition means that the present value of the maximum of the Pontryagin function (the present value of the Hamiltonian) converges to zero when time goes to infinity.
} 
infinite horizon optimization problem. The value of the Hamiltonian function evaluated along the considered trajectories is $H(c, \lambda)=\lambda(t) c(t)=C_{1} C_{2} e^{\rho t}$; it follows that

$$
\lim _{t \rightarrow \infty} e^{-\rho t} H(c, \lambda)=\lim _{t \rightarrow \infty} e^{-\rho t} \lambda(t) c(t)=C_{1} C_{2} \neq 0 .
$$

Hence, no trajectory in the region given by the restrictions $c \geq 1$ and $\lambda>0$ can be optimal.

Consider now trajectories in the region with $0<c<1$ and $\lambda>0$. These trajectories are the solution to

$$
\left\{\begin{array}{l}
\dot{c}=c, \\
\dot{\lambda}=\frac{1}{2}(1-c)+(\rho-1) \lambda .
\end{array}\right.
$$

We show that any trajectory in this region sooner or later enters the region with $c \geq 1$ and $\lambda>0$.

Observe that the equation $\dot{c}=c$ has as solution $c(t)=C_{3} e^{t}$, where $C_{3}$ is a positive constant. Hence, along any trajectory in this region, $c$ is increasing. From (58) follows that if $\lambda=0$, then

$$
\dot{\lambda}=\frac{1}{2}(1-c)>0
$$

for all $c \in(0,1)$. Hence, trajectories in the region with $0<c<1$ and $\lambda>0$ cannot exit this region through the line segment $\{(c, \lambda): c \in(0,1), \lambda=0\}$.

We now show that they also cannot exit through the point $(c, \lambda)=(1,0) \cdot{ }^{43}$ Let $\mathbf{x}=(c, \lambda)$ and $\mathbf{x}_{0}=\left(c_{0}, \lambda_{0}\right)=(1,0)$. Furthermore, let $\mathfrak{F}: D \rightarrow \mathbb{R}^{2}$ be a vector function defined as

$$
\mathfrak{F}(c, \lambda)=(F(c, \lambda), G(c, \lambda)),
$$

where $F$ is defined in (61) and $G$ in (62). ${ }^{44}$ Its domain is $D=\mathbb{R}_{+} \times \mathbb{R} \subset \mathbb{R}^{2}$. We are then looking for a solution to a 2-dimensional nonlinear autonomous dynamical system of the form

$$
\dot{\mathbf{x}}=\mathfrak{F}(\mathbf{x}), \quad \mathbf{x}(0)=\mathbf{x}_{0} .
$$

Take $0<r<1$. Then the set

$$
D_{0}=\overline{B_{r}\left(\mathbf{x}_{0}\right)}=\left\{\mathbf{x} \in D:\left\|\mathbf{x}-\mathbf{x}_{0}\right\| \leq r\right\}
$$

is a neighborhood of $\mathbf{x}_{0}$. First, we show that the restriction of the functions $F$ and $G$

\footnotetext{
${ }^{43}$ If they exited through this point, they would satisfy the transversality conditions as $\dot{\lambda}=0$ for $\lambda=0$ and $c \geq 1$.

${ }^{44}$ Though continuous, functions $F$ and $G$ are not differentiable: $F$ is not differentiable with respect to $\lambda$ at $\lambda=0$, whereas $G$ is not differentiable with respect to $c$ at $c=1$. Using Peano's existence theorem, the continuity of $\mathfrak{F}$ implies that at least one solution to (67) exists. However, continuity is not enough to guarantee uniqueness. An additional condition that needs to be fulfilled to guarantee uniqueness of solutions, at least in some neighborhood of $\mathbf{x}_{0}$, is that $\mathfrak{F}$ is locally Lipschitz in $\mathbf{x}$ at $\mathbf{x}_{0}$.
} 
on $D_{0}$ (a compact subset of $D$ ) is Lipschitz. ${ }^{45}$ Consider first the function

$$
F(c, \lambda)=c+\lambda \chi_{(-\infty, 0]}(\lambda) \frac{\phi^{2} c^{2}}{2}
$$

Write for brevity $\chi=\chi_{(-\infty, 0]}$ and set $h(\lambda)=\chi(\lambda) \lambda$. Then,

$$
|h(\lambda)-h(0)|=|\chi(\lambda) \lambda-0|=|\chi(\lambda) \lambda| \leq|\lambda|=1 \cdot|\lambda-0| .
$$

Hence, $h$ is Lipschitz on $D_{0}$. As on compact sets continuously differentiable functions are Lipschitz, as well as sums and products of Lipschitz functions, it follows that $F$ is Lipschitz on $D_{0}$. Consider now on $D_{0}$ the function

$$
G=(\rho-1) \lambda-\lambda^{2} \chi_{(-\infty, 0]}(\lambda) \frac{\phi^{2} c}{2}+\frac{1-c}{2} \chi_{[0,1)}(c)
$$

The first term of this expression is a linear function; the second term is the function $h$ introduced above times a differentiable function. That the final term is Lipschitz at $c=1$ is demonstrated in the same way as for the function $h$. It follows that $G$ is Lipschitz on $D_{0}$.

We have proved that the functions $F$ and $G$ are locally Lipschitz at $\mathbf{x}_{0}$. Consequently, so is the vector function $\mathfrak{F}$. By the Picard-Lindelöf Theorem, the system (67) has a unique solution in a neighborhood of $\mathbf{x}_{0}$. As this point is on the exit trajectory, no other trajectory can pass through it, in particular no trajectory from the region with $\lambda>0$. As $\dot{c}>0$ in the region with $0<c<1$ and $\lambda>0$, as shown above, all trajectories in this region exit through $\{c=1, \lambda>0\}$ and enter the region with $c \geq 1$ and $\lambda>0$ as $t \rightarrow \infty$. We have already shown that none of these can be optimal.

Moreover, trajectories through points in the set $\{(c, \lambda): c \in(0,1), \lambda=0\}$ satisfy $\dot{\lambda}>0$; they move in the region for which $\lambda>0$ and hence cannot be a part of any optimal trajectory. Due to a one-to-one correspondence between $\lambda$ and $k$ as given in the first part of (21), this set corresponds to the set $\{(c, k): c \in(0,1), k=0\}$ in the state-control representation of the solution. As we have shown, all trajectories leading to these points violate the transversality conditions which every optimal trajectory must necessarily satisfy.

\section{G Proof of Lemma 5}

Assume that there is an optimal investment schedule for which $c \rightarrow 0$ and $k \rightarrow \infty$ as $t \rightarrow \infty$.

\footnotetext{
${ }^{45}$ Simply put, a function $f: D \rightarrow \mathbb{R}^{n}$ is said to be Lipschitz on $B \subseteq D$ if there exists a constant $K>0$ such that $\|f(x)-f(y)\| \leq K\|x-y\|$, for all $x, y \in B$. See Kelley and Peterson (2010), ch. 8, and Sohrab (2003), ch. 4, for the introduction to concepts and precise definitions of terms used in this section.
} 
The instantaneous profit function of the firm reads as

$$
\pi=(1-q-c) q-k^{2} .
$$

As $c \rightarrow 0$ along the trajectories considered, they sooner or later enter the region with positive production, where we have $q=(1-c) / 2$. Substituting this expression into (70), we obtain:

$$
\pi=\frac{(1-c)^{2}}{4}-k^{2} .
$$

As $c \rightarrow 0,(1-c)^{2} / 4$ approaches its upper bound of $1 / 4$. The second term in the above equation, $-k^{2}$, decreases beyond all bounds as $k \rightarrow \infty$. As $t \rightarrow \infty$, there is therefore a time $t_{0}$ such that $\pi(t)=0$ for $t=t_{0}$, and $\pi(t)<0$ for all $t>t_{0}$.

Changing the investment schedule to $k(t)=0$ for all $t \geq t_{0}$ would yield a higher value of total discounted profits $\Pi$, contradicting the assumption.

\section{H Proof of Lemma 6}

We know that the state-control system with zero production is:

$$
\begin{aligned}
\dot{k}(t) & =\rho k(t), \\
\dot{c}(t) & =c(t)(1-\phi k(t)) .
\end{aligned}
$$

At $k=0$, this reduces to: $\dot{k}(t)=0, \quad \dot{c}(t)=c$. Hence, marginal costs increase to infinity along the exit trajectory as $t \rightarrow \infty$. However, as $\lambda=0$ for $c \geq 1$ along the exit trajectory, the transversality condition (17) is satisfied. Observe that along the exit trajectory $k>0$ for $0<c<1$ and $k=0$ for $c \geq 1$. Consequently, it follows from (21) that $\lambda=0$ at $c=1$. Then, from (58) we have that indeed $\lambda=0$ for all $c \geq 1$. Increasing marginal costs along the exit trajectory sooner or later exceed the value of 1 , for which the value of $H(c, \lambda)$ becomes 0 (see (59)). Hence, the transversality condition (16) is satisfied as well.

\section{Proof of Corollary 1}

In this appendix, we prove that the only candidates for an optimal solution curve are the stable path of the saddle-point steady state and the exit trajectory. In particular, we show that any solution curve of the state-control system, given in (26) and (28) and depicted in Figure 3, starting at a point $\left(c_{0}, k_{0}\right)$ with $c_{0}, k_{0}>0$ either (i) ends on the stable path, (ii) ends on the exit trajectory, (iii) gives rise to a control $k(t)$ that goes to infinity, and then satisfies the condition of Lemma 5, or (iv) passes through the line segment $\{(c, k): c \in(0,1), k=0\}$, and is then excluded as an optimal 
solution by Lemma 4. We note that the vector field defined by the state-control system is always locally Lipschitz, and that therefore the theorem of existence and uniqueness of trajectories through a given initial point holds.

There are two situations, determined by the location of the maximum $\left(\frac{1}{2}, k^{*}\right)$ of the $\dot{k}=0$ isocline which is the quadratic function $c \mapsto \frac{\phi}{4 \rho} c(1-c)$. In the first situation $k^{*} \geq 1 / \phi$, in the second $k^{*}<1 / \phi$.

If $k^{*} \geq 1 / \phi$, the state-control space $S=\{(c, k): c>0, k>0\}$ can be partitioned in three regions $S_{1}, S_{2}$, and $S_{3}$. The first set is defined as follows:

$$
S_{1}=\left\{(c, k): 0<c<1, k>k^{*}\right\},
$$

where $k^{*}=\phi /(16 \rho)$ is the maximum of $c \mapsto \frac{\phi}{4 \rho} c(1-c)$. To define the second set, we note that the trajectory $\gamma$ of the state-control space that passes through the point $(c, k)=(1, \phi /(16 \rho))$, when continued backwards in time, necessarily has a second intersection with the line $c=1$. The first of these intersections, as time decreases, is denoted $(c, k)=\left(1, k_{*}\right)$, where $0<k_{*}<1 / \phi$. Let $D$ be the region bounded by $\gamma$ and the line $c=1$. Then,

$$
S_{2}=\{(c, k): c \geq 1, k>0\} \backslash D .
$$

Finally,

$$
S_{3}=S \backslash\left(S_{1} \cup S_{2}\right) .
$$

From the state-control equations

$$
\dot{c}=c(1-\phi k), \quad \dot{k}=\rho k-\frac{\phi}{4} c(1-c),
$$

and the fact that $k^{*}>1 / \phi$, it follows that everywhere in $S_{1}$ we have $\dot{c}<0$ and $\dot{k}>0$. It follows by Lemma 5 that no trajectory in this region can be optimal.

In region $S_{2}$, the state-control equations read as

$$
\dot{c}=c(1-\phi k), \quad \dot{k}=\rho k .
$$

We claim that every trajectory in this region must leave it through the half-line $\ell_{1}=$ $\left\{(c, k): c=1, k \geq k^{*}\right\}$. Note first that the boundary of $S_{2}$ consists of $\ell_{1}$, the curve $\gamma$, the line segment $\ell_{2}=\left\{(c, k): c=1,0<k \leq k_{*}\right\}$, and the half-line $\ell_{3}=\{(c, k):$ $c \geq 1, k=0\}$. As $\gamma$ and $\ell_{3}$ are parts of trajectories of the state-control system, no trajectories can leave $S_{2}$ through them. Moreover, we have $\dot{c} \geq 0$ on $\ell_{2}$, so exit through this part of the boundary is also impossible. The remaining possibilities are to leave through $\ell_{1}$, as claimed, or to remain in $S_{2}$ indefinitely.

To show that the latter alternative is impossible, note that since $\dot{k}=\rho k$, any tra- 
jectory in $S_{2}$ will eventually satisfy $k>2 / \phi$. But then $\dot{c}<-c$, and this implies that eventually $c$ should satisfy $c=1$, leaving the region $S_{2}$ towards $S_{1}$. As no trajectory in $S_{1}$ can be optimal, this now extends to all trajectories in $S_{2}$.

It remains to analyze the trajectories in $S_{3}$. They can leave that region through the line segments $\ell_{2}, \ell_{4}=\{(c, k): 0<c<1, k=0\}$ or $\ell_{5}=\{(c, k): 0<c<1, k=$ $\left.k^{*}\right\}$, as the remaining parts of the boundary are trajectories of the state-control system, or through the point $(1,0)$ on the exit trajectory.

Trajectories leaving through $\ell_{2}$ enter $S_{2}$ and therefore cannot be optimal. As noted above, trajectories leaving through $\ell_{4}$ are excluded by Lemma 4 from optimality. Trajectories leaving through $\ell_{5}$ enter $S_{1}$ and again cannot be optimal. Of all the trajectories leaving $S_{3}$, only those on the exit trajectory are thus candidates for optimal solutions.

It remains to discuss the trajectories that remain in $S_{3}$ for all time. By the PoincaréBendixon theorem, since $S_{3}$ is bounded, the limit set of such a trajectory is either a steady-state point or a closed curve. The latter possibility can be ruled out as the area enclosed by the curve would be invariant (cf. Wagener, 2003). The only steady state in $S_{3}$ that can be approached by a trajectory is the saddle, if $k^{*}>1 / \phi$, or the semistable steady state, if $k^{*}=1 / \phi$, and this shows the result.

If $k^{*}<1 / \phi$, the situation is much simpler. Define in that case

$$
\begin{aligned}
& S_{1}=\{(c, k): 0<c<1, k>1 / \phi\}, \\
& S_{2}=\{(c, k): c \geq 1, k>0\}, \\
& S_{3}=S \backslash\left(S_{1} \cup S_{2}\right) .
\end{aligned}
$$

As above, points in $S_{1}$ cannot be optimal as a consequence of Lemma 5; points in $S_{2}$ eventually end up in $S_{1}$ and are excluded by the same reasoning; and as there are no saddle points in $S_{3}$ and the trajectories leaving through $\ell_{4}$ cannot be optimal, the only remaining candidate is the exit trajectory.

\section{J Proof of Lemma 8}

From (31), we know that the Hamiltonian for $c \geq 1$ is given by:

$$
H(c, k)=k\left(k-\frac{2}{\phi}\right) ;
$$

and from Lemma 7, we know that the comparison of the total discounted profits of each two candidate optimal paths amounts to comparing the values of the respective Hamiltonians in the initial point of each respective path. As the Hamiltonian in the case of zero investment and zero production is zero, the indifference point between the stable path and the exit trajectory in the region with zero production must be the point 
at which the Hamiltonian evaluated along the stable path obtains the value of zero.

$$
\begin{aligned}
& H(c, k)=k\left(k-\frac{2}{\phi}\right)=0 \\
& \Rightarrow k=0 \quad \text { or } \quad k=\frac{2}{\phi}
\end{aligned}
$$

The solution structure (39)-(40) tells us that the stable path in the region with $c \geq 1$ decreases as $t \rightarrow-\infty$, assuming that the stable path covers the respective region. Observe that the derivative of the Hamiltonian with respect to $k$ is $\frac{\partial H(c, k)}{\partial k}=2\left(k-\frac{1}{\phi}\right)$, which is positive for $k>1 / \phi$ and negative for $k<1 / \phi$.

If the stable path enters the region with zero production at all, it enters this region at the point where $k>\frac{1}{\phi}$; this follows directly from equation (28).

The same equation implies that $k$ is decreasing along the stable path as $t \rightarrow-\infty$, assuming the stable path enters the zero-production region. The above conclusions lead us to distinguish three cases. First, if the stable path crosses the boundary line $c=1$ at $k>\frac{2}{\phi}$, then the value of the Hamiltonian is decreasing along the stable path in the region with $c \geq 1$ as $t \rightarrow-\infty$, passes zero at $k=\frac{2}{\phi}$ and is negative afterwards. In this case, the indifference point is the value of the marginal cost $\hat{c}>1$ that corresponds to the point $\left(\hat{c}, \frac{2}{\phi}\right)$ on the stable path. Second, if the stable path reaches $c=1$ at exactly $k=\frac{2}{\phi}$, then the indifference point is $c=1$. Third, for lower values of $k$ on the stable path at $c=1$, the value of the Hamiltonian evaluated along the stable path in the zero-production region is negative, such that the point of indifference (if at all) must be in the region with positive production. 